ARTICLE

\title{
Inositol pyrophosphates promote the interaction of SPX domains with the coiled-coil motif of PHR transcription factors to regulate plant phosphate homeostasis
}

\author{
Martina K. Ried ${ }^{1,7,9}$, Rebekka Wild (10 1,8,9, Jinsheng Zhu ${ }^{1}$, Joka Pipercevic ${ }^{2}$, Kristina Sturm', Larissa Broger ${ }^{1}$, \\ Robert K. Harmel ${ }^{3,4}$, Luciano A. Abriata (1) ${ }^{5}$, Ludwig A. Hothorn ${ }^{6,10}$, Dorothea Fiedler 3,4 , Sebastian Hiller (D) ${ }^{2}$ \&
} Michael Hothorn (1D) ${ }^{1 凶}$

Phosphorus is an essential nutrient taken up by organisms in the form of inorganic phosphate (Pi). Eukaryotes have evolved sophisticated Pi sensing and signaling cascades, enabling them to stably maintain cellular $\mathrm{Pi}$ concentrations. Pi homeostasis is regulated by inositol pyrophosphate signaling molecules (PP-InsPs), which are sensed by SPX domain-containing proteins. In plants, PP-InsP-bound SPX receptors inactivate Myb coiled-coil (MYB-CC) Pi starvation response transcription factors (PHRs) by an unknown mechanism. Here we report that a InsP $\mathrm{P}_{8}$-SPX complex targets the plant-unique CC domain of PHRs. Crystal structures of the CC domain reveal an unusual four-stranded anti-parallel arrangement. Interface mutations in the CC domain yield monomeric PHR1, which is no longer able to bind DNA with high affinity. Mutation of conserved basic residues located at the surface of the $\mathrm{CC}$ domain disrupt interaction with the SPX receptor in vitro and in planta, resulting in constitutive Pi starvation responses. Together, our findings suggest that $\mathrm{Ins}_{8}$ regulates plant $\mathrm{Pi}$ homeostasis by controlling the oligomeric state and hence the promoter binding capability of PHRs via their SPX receptors.

\footnotetext{
${ }^{1}$ Structural Plant Biology Laboratory, Department of Botany and Plant Biology, University of Geneva, 1211 Geneva, Switzerland. ${ }^{2}$ Biozentrum Basel, 4056 Basel, Switzerland. ${ }^{3}$ Leibniz-Forschungsinstitut für Molekulare Pharmakologie, 13125 Berlin, Germany. ${ }^{4}$ Department of Chemistry, Humboldt-Universität zu Berlin, 12489 Berlin, Germany. ${ }^{5}$ Protein production and structure Core Facility, EPFL, 1015 Lausanne, Switzerland. ${ }^{6}$ Institute of Biostatistics, Leibniz University, 30419 Hannover, Germany. ${ }^{7}$ Present address: Leibniz Institute of Plant Biochemistry, 06120 Halle, Germany. ${ }^{8}$ Present address: Institut de Biologie Structurale (IBS), 38044 Grenoble, France. ${ }^{9}$ These authors contributed equally: Martina K. Ried, Rebekka Wild. ${ }^{10}$ Ludwig A. Hothorn is retired.

凶email: michael.hothorn@unige.ch
} 
$\mathrm{P}$ hosphorus is an essential building block for many cellular components such as nucleic acids and membranes. It is essential for energy transfer and storage, and can act as a signaling molecule. Pro- and eukaryotes have evolved intricate systems to acquire phosphorus in the form of inorganic phosphate $(\mathrm{Pi})$, to maintain cytosolic $\mathrm{Pi}$ concentrations and to transport and store $\mathrm{Pi}$ as needed. In green algae and plants, transcription factors have been previously identified as master regulators of $\mathrm{Pi}$ homeostasis and $\mathrm{Pi}$ starvation responses (PSR) ${ }^{1,2}$. Phosphorus starvation response 1 (CrPsr1) from Chlamydomonas and PHOSPHATE STARVATION RESPONSE 1 (AtPHR1) from Arabidopsis were founding members of plant-unique MYB-type coiled-coil (MYB-CC) transcription factors ${ }^{3}$. PHR transcription factors were subsequently characterized as regulators of PSRs in diverse plant species ${ }^{4-6}$. In Arabidopsis, there are 15 MYB-CCs with PHR1 and PHL1 controlling the majority of the transcriptional PSRs ${ }^{7}$. Knockout mutations in Arabidopsis thaliana PHR1 (AtPHR1) result in impaired responsiveness of $\mathrm{Pi}$ starvation induced (PSI) genes and perturbed anthocyanin accumulation, carbohydrate metabolism, and lipid composition ${ }^{2,8,9}$. Overexpression of AtPHR1 causes elevated cellular Pi concentrations and impacts the transcript levels of AtPHO2, which codes for an E2 ubiquitin conjugase involved in PSR, via increased production of its micro RNA miR399d ${ }^{8,10}$. PHR binds to a GNATATNC motif (P1BS), found highly enriched in the promoters of PSI genes and in other cis-regulatory motifs, activating gene expression $^{2,7}$. AtPHR1 is not only implicated in Pi homeostasis but also in sulfate, iron, and zinc homeostasis, as well as in the adaption to high-light stress ${ }^{11-14}$. Moreover, AtPHR1 shapes the plant root microbiome by negatively regulating plant immunity ${ }^{15}$.

AtPHR1 and OsPHR2 have been previously reported to physically interact with stand-alone SPX proteins ${ }^{16-19}$, additional components of PSR in plants ${ }^{17,20-22}$. SPX proteins may regulate PHR function by binding to PHRs under Pi-sufficient condition, keeping the transcription factor from entering the nucleus ${ }^{23-25}$. Alternatively, binding of SPX proteins to PHRs may reduce the ability of the transcription factors to interact with their promoter core sequences ${ }^{18,19,23,24,26}$. Two mechanisms were put forward regarding the regulation of the SPX-PHR interaction in response to changes in nutrient availability: SPX domains were proposed to act as direct Pi sensors, with the SPX-PHR interaction occurring in the presence of millimolar concentrations of $\mathrm{Pi}^{18,19}$. Alternatively, the integrity of the SPX-PHR complex could be regulated by protein degradation. Indeed, SPX degradation via the $26 \mathrm{~S}$ proteasome is increased under Pi starvation ${ }^{23,24,27}$

Fungal, plant, and human SPX domains ${ }^{28}$ have been independently characterized as cellular receptors for inositol pyrophosphates (PP-InsPs), which bind SPX domains with high affinity and selectivity ${ }^{29,30}$. PP-InsPs consist of a fully phosphorylated myo-inositol ring, carrying one or two pyrophosphate groups at the $\mathrm{C} 1$ and/or $\mathrm{C} 5$ position, respectively ${ }^{31}$. In plants, inositol 1,3,4-trisphosphate 5/6-kinase catalyzes the phosphorylation of phytic acid $\left(\mathrm{InsP}_{6}\right)$ to $5 \mathrm{PP}-\mathrm{InsP}_{5}\left(\mathrm{InsP}_{7} \text { hereafter }\right)^{32}$. The diphosphoinositol pentakisphosphate kinases VIH1 and $\mathrm{VIH} 2$ then generate $1,5(\mathrm{PP})_{2}-\mathrm{InsP}_{4}$ (InsP $\mathrm{P}_{8}$ hereafter) from $\mathrm{Ins}_{7} \mathrm{P}^{29,33-35}$. Plant diphosphoinositol pentakisphosphate kinases have been genetically characterized to play a role in jasmonate perception and plant defense responses ${ }^{34}$ and, importantly, in nutrient sensing in Chlamydomonas ${ }^{36}$ and Arabidopsis ${ }^{29,35}$. vih1 vih2 double mutants lack the PP-InsP messenger Ins $\mathrm{P}_{8}$, over accumulate $\mathrm{Pi}$, and show constitutive PSI gene expression ${ }^{29,35}$. A vih1 vih2 phr1 phl1 quadruple mutant rescues the vih1 vih2 seedling phenotypes and displays wild-type-like Pi levels, suggesting that VIH1, VIH2, PP-InsPs, and PHRs are part of a common signaling pathway ${ }^{35}$. In line with, the AtSPX1-AtPHR1 interaction is reduced in vih1 vih2 mutant plants when compared to wild type ${ }^{29}$. Thus, biochemical and genetic evidence implicates $\mathrm{InsP}_{8}$ in the formation of a SPX-PHR complex ${ }^{29,35}$.

Cellular Ins $\mathrm{P}_{8}$ pools are regulated by nutrient availability at the level of the VIH enzymes themselves. Plant VIH1 and VIH2, and diphosphoinositol pentakisphosphate kinases from other organisms are bifunctional enzymes, with an $\mathrm{N}$-terminal kinase domain that generates Ins $_{8}$ from $\mathrm{Ins}_{7}$ and a C-terminal phosphatase domain that hydrolyzes $\mathrm{InsP}_{8}$ to $\mathrm{InsP}_{7}$ and $\mathrm{InsP}_{6} \mathrm{P}^{35,37,38}$. The relative enzymatic activities of the two domains are regulated in the context of the full-length enzyme: under Pi starvation, cellular ATP levels are reduced, leading to a reduction of the VIH kinase activity and a reduction of Ins $\mathrm{P}_{8}{ }^{29,35}$. Pi itself acts as an allosteric regulator of the phosphatase activity ${ }^{35,37}$. Thus, under $\mathrm{Pi}$ sufficient growth conditions, $\mathrm{Ins}_{8}$ accumulates and triggers the formation of a SPX-Ins $\mathrm{P}_{8}-\mathrm{PHR}$ complex. Under Pi starvation, $\mathrm{InsP}_{8}$ levels drop and the complex dissociates ${ }^{29}$.

How the $\mathrm{InsP}_{8}$-bound SPX receptor inactivates PHR function remains to be understood at the mechanistic level. It has been previously reported that AtPHR1 binds P1BS as a dimer ${ }^{2}$. Addition of SPX domains reduces the DNA-binding capacity of PHRs as concluded from electrophoretic mobility shift assays (EMSAs) ${ }^{18,19,23}$. Qi et al. ${ }^{26}$ reported that AtPHR1 recombinantly expressed as a maltose-binding protein (MBP) fusion protein forms monomers in solution and binds DNA. This process can be inhibited by preincubating the recombinant transcription factor with AtSPX1 in the presence of high concentrations of $\operatorname{InsP}_{6}{ }^{26}$. A recent crystal structure of the AtPHR1 MYB domain in complex with a promoter core fragment supports a dimeric binding mode of MYB-CC transcription factors ${ }^{39}$. Here we investigate the oligomeric state of PHRs, their DNA-binding kinetics, and the targeting mechanism of the interacting SPX receptors.

\section{Results}

PP-InsPs trigger AtSPX1-AtPHR1 complex formation in yeast. The interaction of AtSPX1 with AtPHR1 has been previously characterized in yeast two-hybrid assays ${ }^{19}$. We reproduced the interaction of full-length AtPHR1 and AtSPX1 (Fig. 1a), and verified that all four stand-alone AtSPX proteins (AtSPX1-4) interact with a AtPHR1 fragment (AtPHR1 226-360) that contains the MYB domain and the CC domain in yeast (Supplementary Fig. 1a). This is in line with previous findings, reporting interaction of SPX domains with larger PHR fragments also containing the MYB and CC domains (AtSPX1-AtPHR1 ${ }^{208-362}$ and OsSPX1/2-OsPHR2 $\left.231-426\right)^{18,19}$.

We next tested whether the SPX-PHR interactions observed in yeast are mediated by endogenous PP-InsPs. The putative PP-InsPbinding surface in AtSPX1 was mapped by homology modeling, using the previously determined Chaetomium thermophilum Gde1-InsP ${ }_{6}$ complex structure (PDB-ID 5IJJ) as template ${ }^{30}$. We replaced putative $\mathrm{PP}$-InsP-binding residues from the previously identified phosphate binding cluster (PBC: AtSPX1Y25, K29, K139) and Lysine (K) surface cluster (KSC: AtSPX1 K136, K140, K143) ${ }^{30}$ with alanines (Supplementary Fig. 1b). The resulting AtSPX1 ${ }^{\mathrm{PBC}}$ and AtSPX1 ${ }^{\mathrm{KSC}}$ mutant proteins failed to interact with AtPHR1 ${ }^{226-360}$ in yeast two-hybrid assays, whereas mutation of a conserved lysine residue outside the putative PP-InsP-binding site (structural control, AtSPX1 ${ }^{\mathrm{K} 81}$ ) to alanine had no effect (Supplementary Fig. 1b). We next deleted the known yeast PP-InsP kinase Vip1, which converts Ins $_{6}$ to $1 \mathrm{PP}-\mathrm{InsP}_{5}$ and $\mathrm{InsP}_{7}$ to $\mathrm{InsP}_{8}$, or the PP-InsP kinase Kcs1, which converts Ins $\mathrm{P}_{6}$ to $\mathrm{InsP}_{7}$ and $1 \mathrm{PP}-\mathrm{InsP}_{5}$ to Ins $_{8}{ }^{40,41}$ (Supplementary Fig. 1c, d). We found that deletion of either kinase reduced the interaction between wild-type AtSPX1 and AtPHR1 226-360 (Supplementary Fig. 1c). The interaction between the plant brassinosteroid receptor kinase BRI1 and the inhibitor protein BKI1, known to occur independently of 


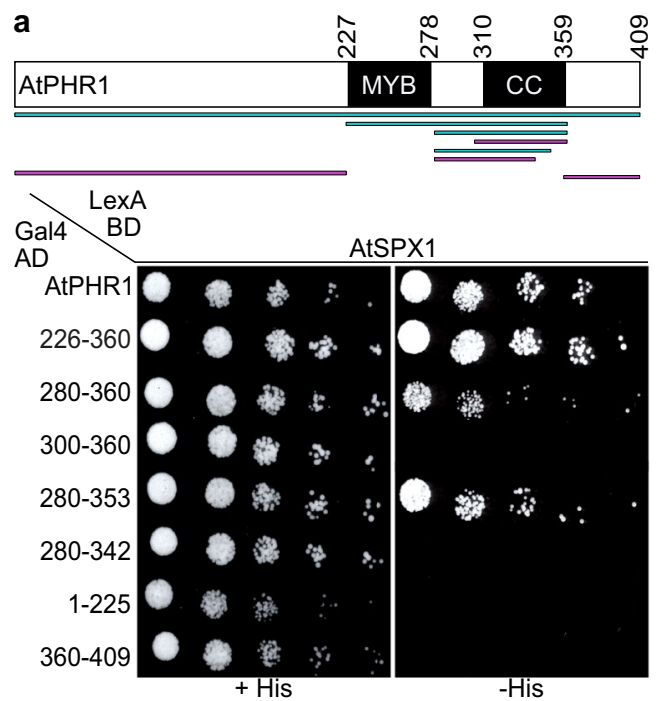

C

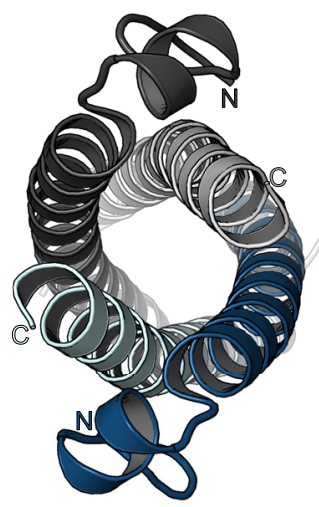

d
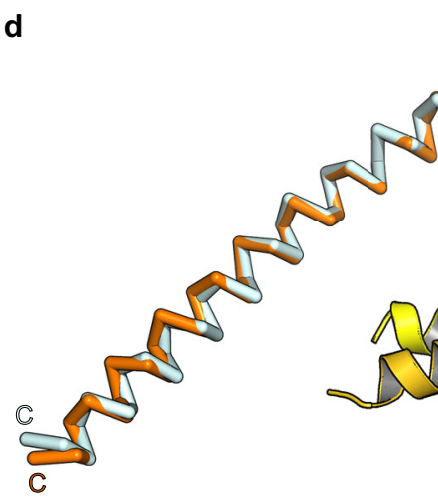<smiles>C1=CC2=CC=CC2=C1</smiles>
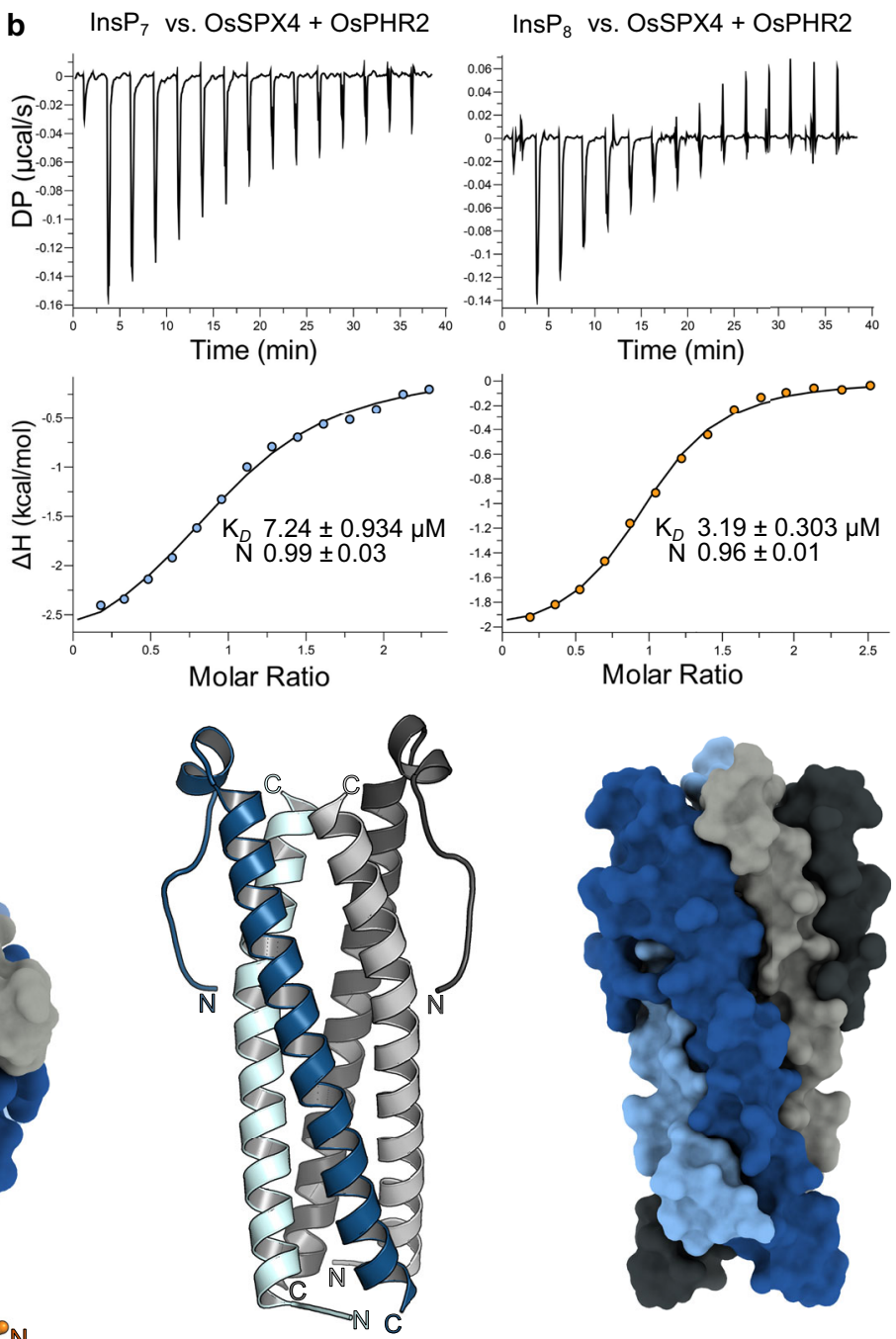<smiles>CC(C)CCNc1ccccc1</smiles>

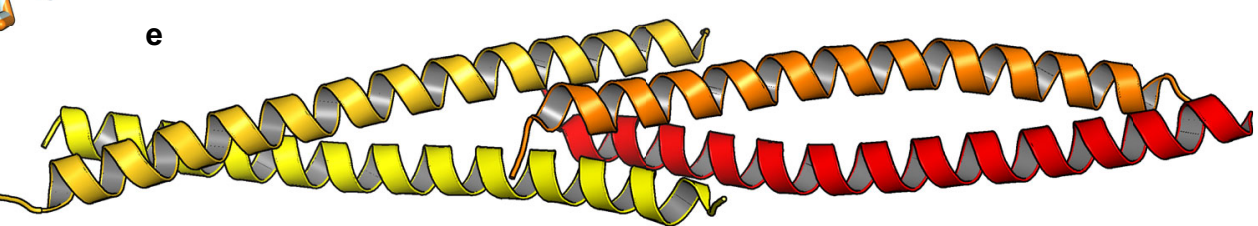

Fig. 1 AtSPX1 recognizes the AtPHR1 coiled-coil domain that crystallizes as a tetramer. a Yeast co-expressing different AtPHR1 deletion constructs fused to the Gal4-activation domain (AD; prey) and full-length wild-type AtSPX1 fused to the LexA-binding domain (BD; bait) were grown on selective SD medium supplemented with histidine (+His; co-transformation control) or lacking histidine (-His; interaction assay) to map a minimal fragment of AtPHR1 sufficient for interaction with AtSPX1. Shown are serial dilutions from left to right. A schematic overview of the tested interacting (in cyan) and non-interacting (in magenta) AtPHR1 fragments is shown alongside (MYB, MYB-DNA-binding domain; CC, coiled-coil domain). b Isothermal titration calorimetry assays of $\operatorname{InsP}_{7}\left(400 \mu \mathrm{M} 5 \mathrm{PP}-\mathrm{InsP}_{5}\right.$; left panel) and InsP $\left(500 \mu \mathrm{M} 1,5(\mathrm{PP})_{2}\right.$-InsP ; right panel) binding to OsSPX4-OsPHR2 (30 $\left.\mu \mathrm{M}\right)$, respectively. Raw heats per injection are shown in the top panel and the bottom panel represents the integrated heats of each injection, fitted to a one-site binding model (solid line). The insets show the dissociation constant $\left(K_{\mathrm{D}}\right)$ and binding stoichiometry $(\mathrm{N})$ ( \pm fitting error). c Ribbon and surface diagrams of the AtPHR1 CC four-stranded anti-parallel tetramer. Helices contributing to the dimer interface are shown in light and dark blue, respectively. Corresponding, symmetry-related helices completing the tetramer are shown in light and dark gray. d Structural superposition of two AtPHR1 core CC helices ( $C_{a}$ trace, in light blue) and ScCtp1 (PDB-ID $4 \times 01$, in orange) ${ }^{44}$. R.m.s.d. is $\sim \AA$ comparing 45 corresponding $C_{a}$ atoms. e Ribbon diagram of the ScCtp1 dimer-of-dimers CC domain, with contributing helices colored from yellow to red. 
PP-InsPs ${ }^{42}$, was not affected in either $\Delta$ vip1 or $\Delta \mathrm{kcs} 1$ mutants (Supplementary Fig. 1c).

Using quantitative isothermal titration calorimetry (ITC)binding assays, we have previously determined dissociation constants $\left(K_{\mathrm{D}}\right)$ for $\mathrm{InsP}_{6}$ and $\mathrm{InsP}_{7}$ binding to a OsSPX4-OsPHR2 complex to be $\sim 50$ and $\sim 7 \mu \mathrm{M}$, respectively ${ }^{30}$. A side-by-side comparison of $\operatorname{InsP}_{7}$ and $\mathrm{InsP}_{8}$ binding to OsSPX4-OsPHR2 by ITC revealed dissociation constants of $\sim 7$ and $\sim 3 \mu \mathrm{M}$, respectively (Fig. 1b). Taken together, the SPX-PHR interaction is mediated by PP-InsPs, with the bona fide $\mathrm{Pi}$ signaling molecule $\mathrm{InsP}_{8}$ being the preferred ligand in vitro.

AtSPX1 interacts with a unique four-stranded coiled-coil domain in AtPHR1. We next mapped the SPX-PP-InsP-binding site in AtPHR1 to a fragment (AtPHR1 $280-353)$, which comprises the CC domain and a 30-amino acid spanning N-terminal extension, in yeast two-hybrid experiments (Fig. 1a). We sought to crystallize an AtSPX1-PP-InsP-AtPHR1 complex either in the pre- or absence of P1BS fragments. We obtained crystals of a putative AtSPX1-InsP ${ }_{8}-$ AtPHR1 $^{280-360}$ complex diffracting to $2.4 \AA$ resolution and solved the structure by molecular replacement, using isolated SPX domain structures as search models ${ }^{30}$. Iterative cycles of model building and crystallographic refinement yielded, to our surprise, a well-refined model of AtPHR $1^{280-360}$ only (see "Methods"). Analysis with the program PISA revealed the presence of a crystallographic tetramer in which four long $\alpha$ helices fold into an unusual anti-parallel four-stranded coiled-coil (Fig. 1c). AtPHR1 ${ }^{280-360}$ residues $292-356$ and $310-357$ are visible in the electron density maps from chain $A$ and $B$, respectively. Residues $292-311$ in chain A fold into a protruding loop region that harbors a small $\alpha$-helix, and appear disordered in chain B (Fig. 1c). The anti-parallel a-helices in AtPHR1 closely align with a root mean square deviation (r.m.s.d.) of $\sim 0.5 \AA$ comparing 45 corresponding $\mathrm{C}_{\mathrm{a}}$ atoms. Structural homology searches with the program DALI ${ }^{43}$ returned different coiled-coil structures, with a monomer of the tetrameric coiled-coil domain of the yeast transcription factor Ctp1 representing the closest hit (DALI $Z$-score 5.9, r.m.s.d. is $\sim 1 \AA$ comparing 45 corresponding $\mathrm{C}_{\mathrm{a}}$ atoms) (Fig. 1d) ${ }^{44}$. However, no anti-parallel four-stranded coiled-coil domain with structural similarity to AtPHR1 was recovered, with, for example, the Ctp1 dimer-of-dimers domain having a very different configuration (Fig. 1e $)^{44}$.

We next assessed the oligomeric state of AtPHR ${ }^{280-360}$ using size-exclusion chromatography coupled to right-angle light scattering (SEC-RALS) and determined an apparent molecular weight of $\sim 37.5 \mathrm{kDa}$, thus confirming that the isolated AtPHR1 CC forms tetramers in solution (theoretical molecular weight of the monomer is $\sim 9.5 \mathrm{kDa}$ ) (Fig. 2a). Two additional crystal structures of AtPHR $1^{280-360}$ obtained in different crystal lattices all revealed highly similar tetrameric arrangements (Supplementary Fig. 2 and Supplementary Table 5). Although we found purified full-length AtPHR1 to be too unstable for SEC-RALS analysis, full-length OsPHR2 formed a tetramer in solution (Supplementary Fig. 3). In contrast, untagged AtPHR1222-358, which comprises the CC and the MYB domains only, runs as a dimer (Fig. 2a, b black traces), in agreement with earlier reports ${ }^{2}$.

Mutations in the CC domain abolish AtPHR1 oligomerization and DNA binding in vitro. The observed oligomeric state differences between full-length OsPHR2, AtPHR1280-360 (CC), and AtPHR1 $1^{222-358}$ (MYB-CC) prompted us to investigate the putative dimer- and tetramerization interfaces in our AtPHR1 structures with the program PISA ${ }^{45}$. We found the dimerization $\left(\sim 1400 \AA^{2}\right.$ buried surface area) and the tetramerization $\left(\sim 1900 \AA^{2}\right.$ buried surface area) interfaces to be mainly formed by hydrophobic interactions (Supplementary Fig. 4a, b). Both interfaces are further stabilized by hydrogen bond interactions and several salt bridges (Supplementary Fig. 4a, b). Importantly, all contributing amino-acids represent sequence fingerprints of the plant-unique MYB-CC transcription factor subfamily and are highly conserved among different plant species (Supplementary Fig. 4c). We identified residues specifically contributing to the formation of a CC dimer (Olig1: AtPHR $1^{\mathrm{L} 319}$, AtPHR $1^{\mathrm{I} 333}$, AtPHR $1^{\mathrm{L} 337}$, shown in cyan in Fig. 2 and Supplementary Fig. 4) or tetramer (Olig2: AtPHR1 ${ }^{\mathrm{L} 317}$, AtPHR1 ${ }^{\mathrm{L} 327}$, AtPHR $1^{\text {I341 }}$, shown in dark orange in Fig. 2 and Supplementary Fig. 4) in our different CC structures (Supplementary Table 5). We replaced these residues by asparagine to generate two triple mutants in AtPHR1 $1^{222-358}$ and AtPHR ${ }^{280-360}$, respectively. We found in SEC-RALS assays that both mutant combinations dissolved AtPHR1 ${ }^{280-360}$ tetramers and AtPHR1 $222-358$ dimers into monomers, respectively (Fig. 2a, b).

Analysis of the secondary structure content of wild-type AtPHR ${ }^{280-360}$ using circular dichroism (CD) spectroscopy revealed a $100 \%$ a-helical protein (Supplementary Fig. 5a), in agreement with our structural model of the AtPHR1 CC domain (Fig. 1c). In contrast, we estimated the secondary structure content of the Olig1 and Olig2 mutant proteins to be $\sim 50 \%$ ahelical and $\sim 50$ random coil (Supplementary Fig. 5a). The CD melting spectrum for wild-type AtPHR ${ }^{280-360}$ indicated the presence of a well-folded protein with a melting temperature $\left(T_{\mathrm{m}}\right)$ of $\sim 50{ }^{\circ} \mathrm{C}$, while we could not reliably determine $T_{\mathrm{m}}$ 's for the Olig1 and Olig2 mutant proteins (Supplementary Fig. 5b). We conclude that mutation of either AtPHR $1^{\mathrm{L} 319}$, AtPHR $1^{\mathrm{I} 333}$, AtPHR $1^{\mathrm{L} 337}$ or AtPHR1 $1^{\mathrm{L} 317}$, AtPHR1 $1^{\mathrm{L} 327}$, AtPHR1 $1^{\mathrm{I} 341}$ to asparagine disrupts the tetrameric coiled-coil domain of AtPHR1 and affects the structural integrity of the contributing a-helix.

It has been recently reported that the AtPHR1 MYB domain associates with its target DNA as a dimer ${ }^{39}$. We thus studied the capacity of AtPHR1 $1^{222-358}$ oligmerization mutants to interact with the P1BS in qualitative EMSA and quantitative grating-coupled interferometry (GCI) assays. AtPHR1 ${ }^{222}$ - 358 Olig1 could still interact with the P1BS in EMSAs indistinguishable from wild type (Fig. 2c). However, AtPHR1222 - 358 Olig1 and AtPHR1222 - 358 Olig2 bound a biotinylated P1BS immobilized on the GCI chip with $\sim 20$-fold reduced affinity when compared to the wild-type control (Fig. 2d-f). Together, our experiments suggest that PHR1 may exist as a tetramer or dimer in solution, and that disruption of its plant-unique CC domain interface reduces the capacity of the transcription factor to bind its DNA recognition site.

CC surface mutations abolish PHR-SPX interactions but do not interfere with DNA binding in vitro. We next sought to identify the binding site for SPX in the PHR CC domain. In our structures, a conserved set of basic residues maps to the surface of the four CC helices (shown in magenta in Fig. 3a and Supplementary Fig. 4c). A similar set of surface-exposed basic residues has been previously found to form the binding site for PP-InsPs in various SPX receptors ${ }^{30}$. Mutation of AtPHR1 ${ }^{\mathrm{K} 325}$, AtPHR $1^{\mathrm{H} 328}$, and AtPHR1 ${ }^{\mathrm{R} 335}$, but not of AtPHR1 ${ }^{\mathrm{K} 308}$, AtPHR1 ${ }^{\mathrm{R} 318}$, and AtPHR1 ${ }^{\mathrm{R} 340}$ to alanine, disrupted the interaction of AtPHR1 with AtSPX1 in yeast (Fig. 3b). We simultaneously mutated the residues corresponding to AtPHR1 ${ }^{\mathrm{K} 325}$, AtPHR $1^{\mathrm{H} 328}$, and AtPHR $1^{\mathrm{R} 335}$ to alanine in OsPHR2 (OsPHR2 $\left.2^{\mathrm{KHR} / \mathrm{A}}\right)$. The mutant transcription factor showed no detectable binding to OsSPX4-InsP $\mathrm{P}_{7}$ in quantitative ITC assays, but maintained the ability to bind the P1BS (Fig. 3c, d). In line with this, mutation of the KHR motif does not alter the oligomeric state of AtPHR $1^{280-360}$ as concluded from SEC-RALS experiments (Fig. 2a magenta traces). 
a
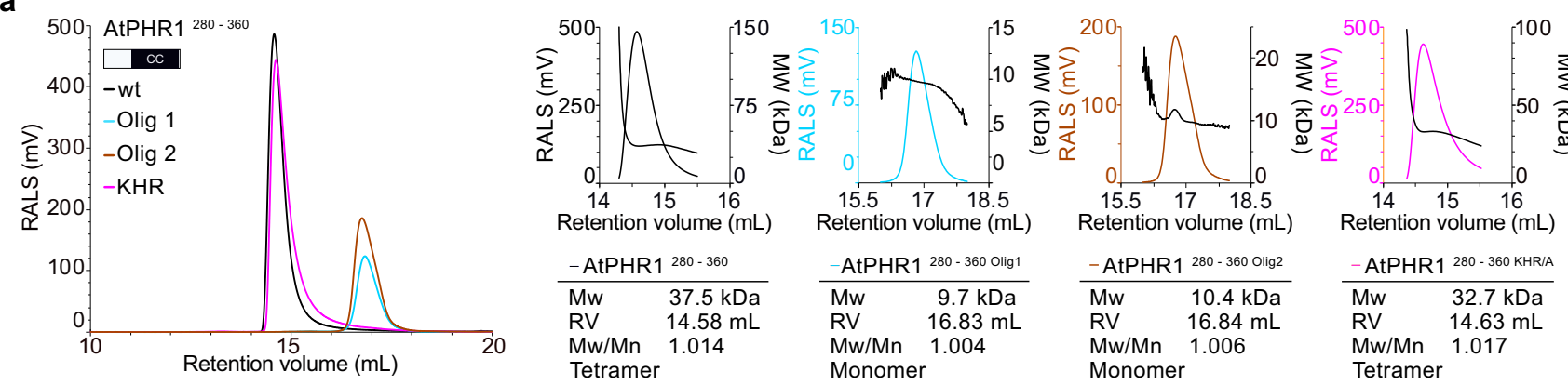

b

\begin{tabular}{|c|c|c|c|}
\hline \multicolumn{2}{|c|}{-AtPHR1 $280-360$} & \multicolumn{2}{|c|}{ AtPHR1 280 - 360 Olig1 } \\
\hline $\mathrm{Mw}$ & $37.5 \mathrm{kDa}$ & $\mathrm{Mw}$ & $9.7 \mathrm{kDa}$ \\
\hline RV & $14.58 \mathrm{~mL}$ & RV & $16.83 \mathrm{~mL}$ \\
\hline Mw/Mn & 1.014 & $\mathrm{Mw} / \mathrm{Mn}$ & 1.004 \\
\hline Tetrame & & Monome & \\
\hline
\end{tabular}
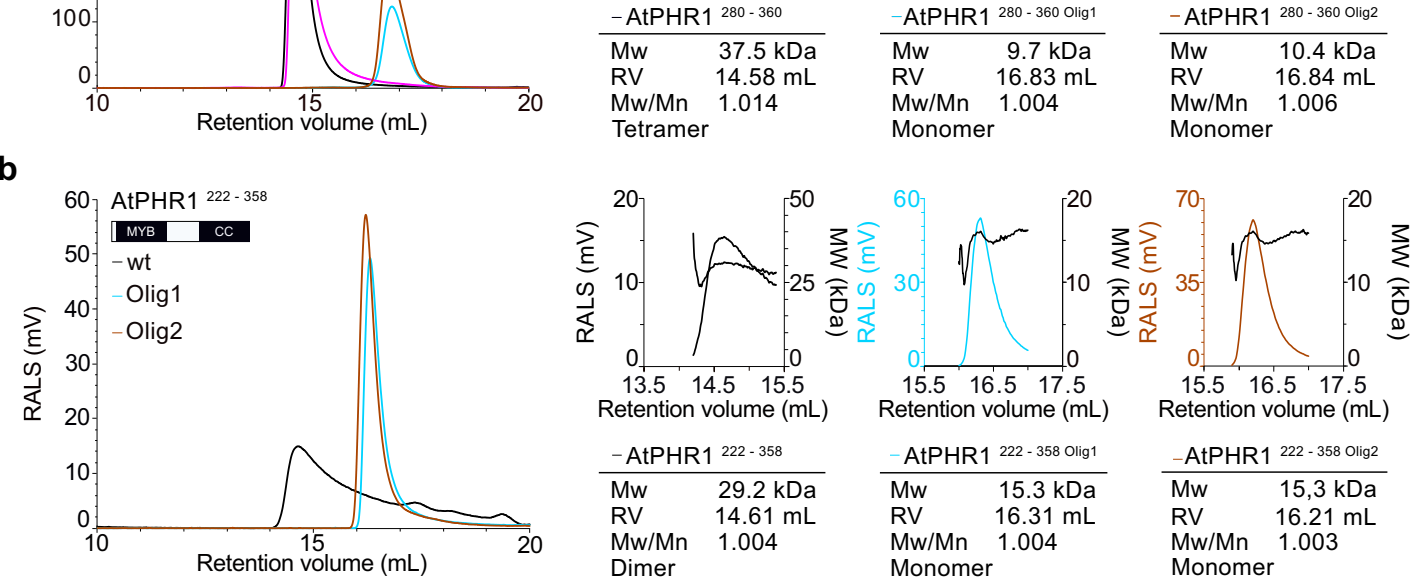

Dimer

Monomer

\begin{tabular}{ll}
-AtPHR1 & 222 - 358 olig2 \\
\hline Mw & $15,3 \mathrm{kDa}$ \\
$\mathrm{RV}$ & $16.21 \mathrm{~mL}$ \\
Mw/Mn & 1.003
\end{tabular}

$\circ$

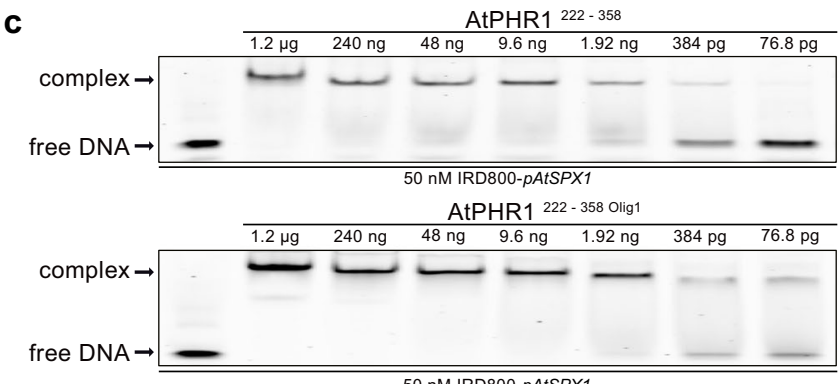

e
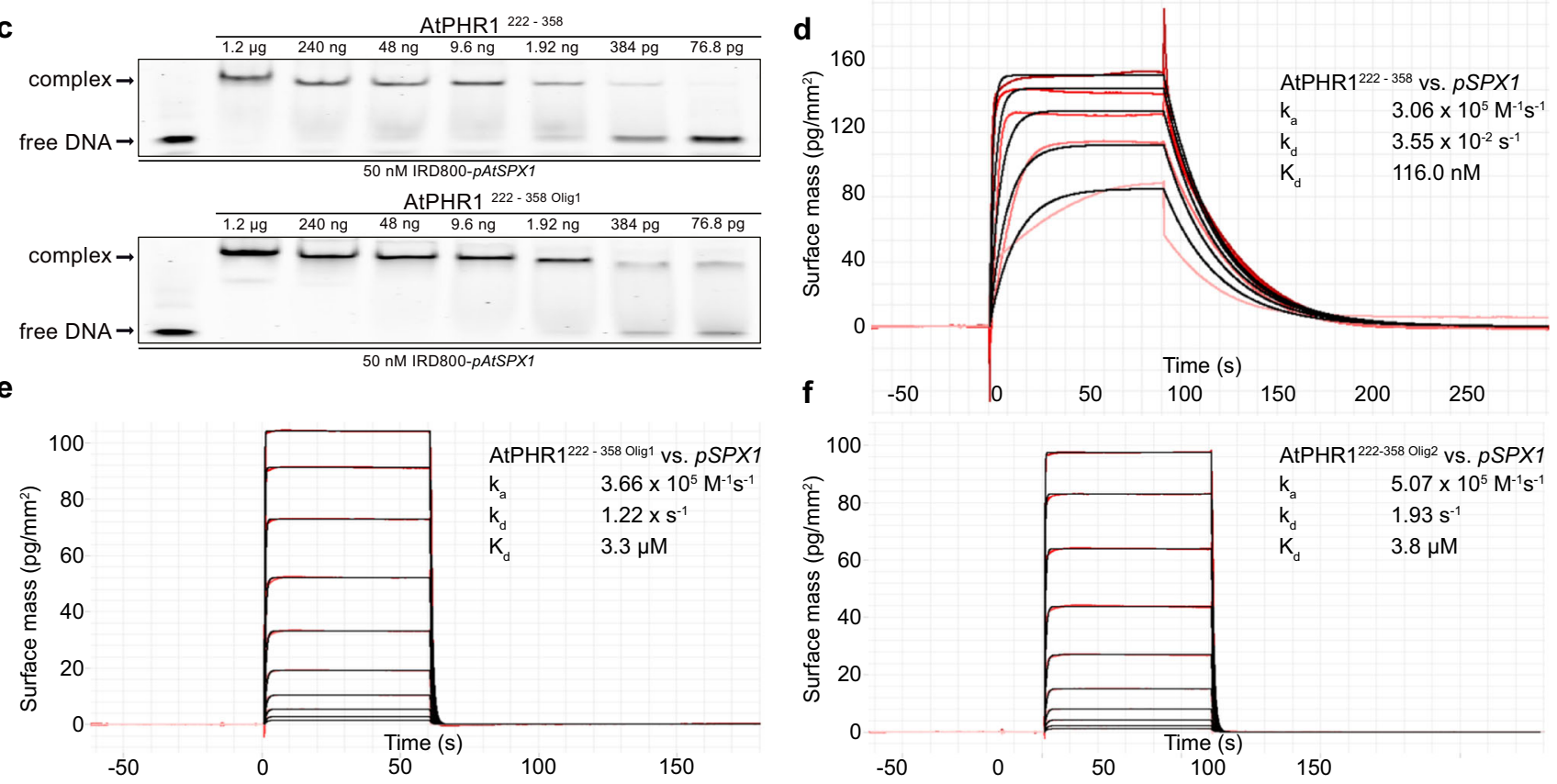

Fig. 2 Mutations in the AtPHR1 coiled-coil domain impair oligomerisation and DNA binding. a Analytical size-exclusion chromatography traces of wildtype AtPHR1 CC (wt, black line), AtPHR1280-360 Olig1 (Olig1, cyan), AtPHR1280-360 Olig2 (Olig2, orange), and of AtPHR1280-360 KHR/A (KHR, magenta). The corresponding right-angle light scattering (RALS) traces are shown alongside, the molecular masses are depicted by a black line. Table summaries provide the molecular weight $(\mathrm{Mw})$, retention volume (RV), dispersity $(\mathrm{Mw} / \mathrm{Mn})$, and the derived oligomeric state of the respective sample. $\mathbf{b}$ Analysis of AtPHR1 MYB-CC (AtPHR1222-358) as described in a. c Qualitative comparison of the interaction of AtPHR1222-358 (upper panel) or AtPHR1222-358 Olig1 (lower panel) binding to IRD800-pAtSPX1 in electrophoretic mobility shift assays. Experiment was performed twice with similar results. d-f Quantitative comparison of the interaction of AtPHR1222-358, AtPHR1 222-358 Olig1, or AtPHR12 22-358 Olig2 with pSPX1 by grating-coupled interferometry (GCI).

Sensorgrams show raw data (red lines) and their respective fits (black lines). Table summaries provide the derived association rate $\left(k_{\mathrm{a}}\right)$, the dissociation rate $\left(k_{d}\right)$, and the dissociation constant $\left(K_{d}\right)$.

Using nuclear magnetic resonance (NMR) spectroscopy, we next tested whether the KHR motif in AtPHR1 is directly involved in PP-InsP ligand recognition. We titrated InsP $\mathrm{P}_{8}$ into ${ }^{15} \mathrm{~N},{ }^{2} \mathrm{H}$-labeled AtPHR1 ${ }^{280-360}$, and recorded TROSY spectra using potassium phosphate $(\mathrm{KPi})$ as control. Five backbone amide moieties exhibited chemical shift perturbations in the presence of
Ins $\mathrm{P}_{8}$ but not in the presence of the KPi control. We acquired titration spectra using increasing concentrations of $\mathrm{InsP}_{8}$ and estimated dissociation constants for $\mathrm{InsP}_{8}$ based on three representative peaks (Supplementary Fig. 6). The derived dissociation constant is in the millimolar range and saturation could not be reached in the available concentration range 
a

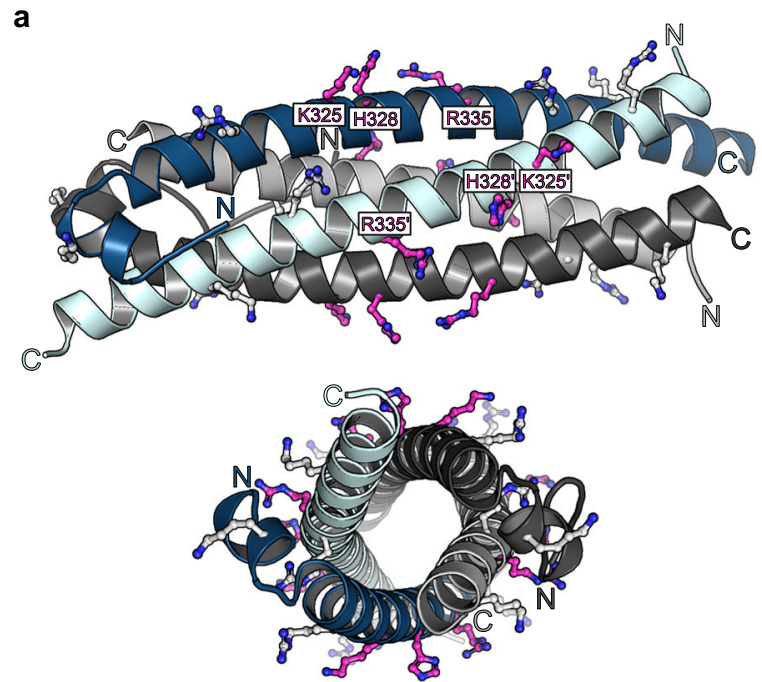

C

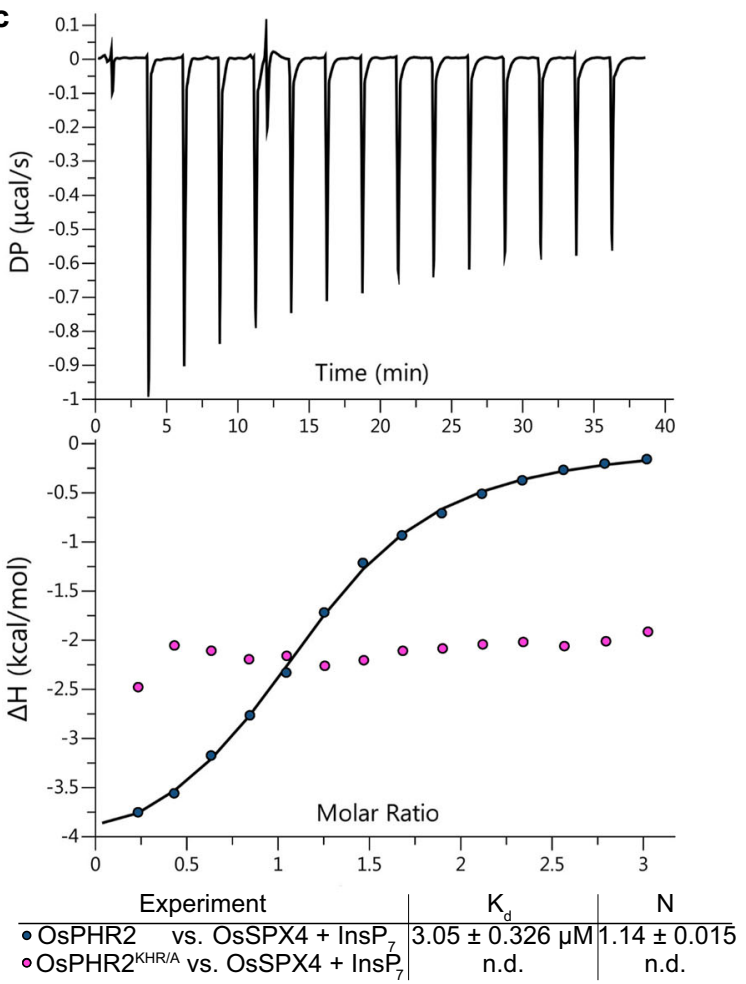

b

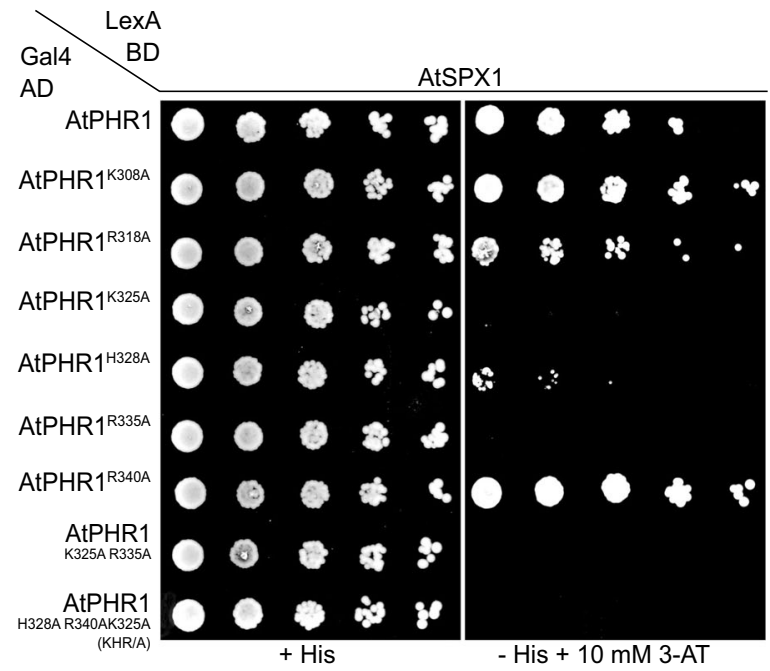

d
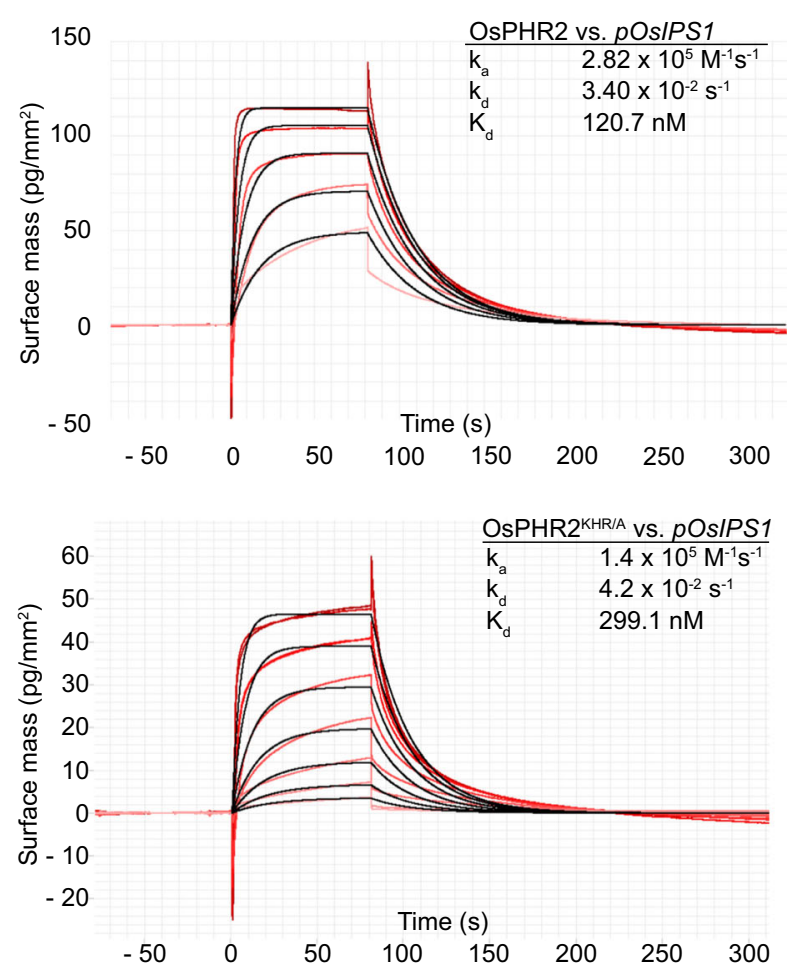

Fig. 3 The KHR motif at the surface of the PHR coiled-coil domain is required for the interaction with SPX domains. a Ribbon diagram of the AtPHR1 CC domain with conserved basic residues located at the surface of the domain shown in bonds representation. The KHR motif (AtPHR1 K325, AtPHR1 ${ }^{\mathrm{H} 328}$, AtPHR1R335) is highlighted in magenta. b Mutational analysis of the basic residues in AtPHR1 CC. Yeast co-expressing AtPHR1226-360 variants in which surface-exposed basic residues have been replaced with alanine fused to the Gal4-AD (prey) and AtSPX1 fused to the LexA-BD (bait) were grown on selective SD medium supplemented with histidine (+His; co-transformation control) or lacking histidine and supplemented with 10 mM 3-amino-1,2,4triazole (3-AT) (-His + $10 \mathrm{mM}$ 3-AT; interaction assay) to identify residues required for interaction with AtSPX1 in yeast two-hybrid assays. Shown are serial dilutions from lift to right. c Isothermal titration calorimetry (ITC) assay of wild-type OsPHR2 and OsPHR2 KHR/A (300 $\mu$ M) vs. OsSPX4 (20 $\mu \mathrm{M})-5 \mathrm{PP}-\operatorname{InsP}_{5}(100 \mu \mathrm{M})$. Raw heats per injection are shown in the top panel, the bottom panel represents the integrated heats of each injection, fitted to a one-site binding model (solid line). The insets show the dissociation constant $\left(K_{\mathrm{D}}\right)$ and binding stoichiometry $(N)$ ( \pm fitting error, n.d. no detectable binding). d Quantitative comparison of the interaction of OsPHR2 (top panel) or OsPHR2KHR/A (bottom panel) with pOsIPS1 by GCI. Sensorgrams show raw data (red lines) and their respective fits (black lines). The insets show summarize association rates $\left(k_{\mathrm{a}}\right)$, dissociation rates $\left(k_{\mathrm{d}}\right)$, and the dissociation constant $\left(K_{d}\right)$ of the respective sample.

(Supplementary Fig. 6). We next repeated the same set of experiments using the AtPHR1 ${ }^{\mathrm{KHR} / \mathrm{A}}$ mutant protein, located the same peaks in the transverse relaxation-optimized spectroscopy (TROSY) spectra and estimated similar dissociation constants (Supplementary Fig. 6).
Taken together, three highly conserved basic residues located at the surface of the PHR coiled-coil domain are critical for the interaction with the PP-InsP-bound SPX receptor (Supplementary Fig. 4c). The NMR titration experiments suggest that AtPHR1 does not contribute to the specific binding of InsP $\mathrm{P}_{8}$ and 
that the low affinity interaction between the CC domain and $\mathrm{InsP}_{8}$ does not involve the KHR motif (Supplementary Fig. 6).

Mutation of the AtPHR1 KHR motif impairs AtSPX1 binding and Pi homeostasis in planta. We next tested whether mutation of the SPX-binding site in AtPHR1 can modulate its function in Pi homeostasis in Arabidopsis. We expressed wild-type and pointmutant versions of AtPHR1 carrying an N-terminal FLAG tag under the control of its native promoter in a phr1-3 loss-offunction mutant ${ }^{8}$. At seedling stage, we found that AtPHR1 single, double, and triple point mutations complemented the previously characterized Pi deficiency phenotype of phr1-32,8. (Fig. 4a and Supplementary Fig. 7a). After transferring the seedlings to soil, variable growth phenotypes became apparent 21 days after germination (DAG) (Supplementary Fig 7b). From three independent lines per genotype, we selected one line each showing similar AtPHR1 transcript levels for all experiments shown in Fig. 4 (Fig. 4 and Supplementary Fig. 8a). Comparing these lines, we found that AtPHR1 ${ }^{\mathrm{K} 325 \mathrm{~A}, \mathrm{R} 335 \mathrm{~A}}$ double and AtPHR 1 K325A,H328A,R335A triple mutants, but not the single mutants, displayed severe growth phenotypes, with the triple mutant showing the strongest defects (Fig. 4a). We next determined cellular Pi levels in all independent lines and found that (i) $\mathrm{Pi}$ levels are positively correlated with AtPHR1 expression levels (Supplementary Fig. 8a), that (ii) all AtPHR1 mutant proteins tested accumulate Pi to significantly higher levels when compared to wild type and phr1-3, and that (iii) the

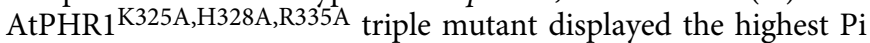
levels (Fig. $4 \mathrm{~b}$ and Supplementary Fig. 8b-d). In line with this, PSI gene expression is misregulated in AtPHR $1^{\mathrm{K} 325 \mathrm{~A}, \mathrm{R} 335 \mathrm{~A}}$ double

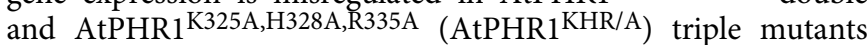
(Fig. 4c). In co-immunoprecipitation assays in Nicotiana benthamiana and in Arabidopsis, we found the interaction of

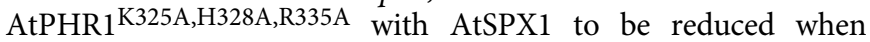
compared to wild-type AtPHR1 (Fig. 4d and Supplementary Fig. 8).

We next studied the genetic interaction between PHR1 and $V I H 1 / 2$. As previously reported, the severe phenotypes of vih1-2 vih2-4 seedlings are partially rescued in the phr1 phl1 vih1-2 vih24 quadruple mutant, suggesting that $\mathrm{VIH1} / 2$-generated $\mathrm{InsP}_{8}$ regulates the activity of PHR1 and PHL1 by promoting the binding of SPX receptors ${ }^{29,35}$. We performed the orthogonal genetic experiment, by complementing the phrl phll mutant with AtPHR1 KHR/A expressed under the control of the AtPHR1 promoter and carrying a $\mathrm{N}$-terminal enhanced green fluorescent protein (eGFP) tag (Fig. 4e, see "Methods"). The complemented lines displayed intermediate growth phenotypes and constitutive PSI gene expression (Fig. 4e, f). Thus, SPX-InsP $P_{8}$ mediated regulation of PHR1 and PHL1 has to be considered one of several PP-InsP regulated processes affected in the vih1-2 vih2-4 mutant. Together, our in vivo experiments reveal that SPX receptors interact with the CC domain of AtPHR1 via the surface-exposed Lys 325 , His 328 , and Arg335, and that this interaction negatively regulates PHR activity and PSRs.

\section{Discussion}

PHR transcription factors have been early on recognized as central components of the PSR in green algae and in plants, directly regulating the expression of PSI genes ${ }^{1,2,7}$. In Arabidopsis and in rice $s p x$ mutants of then unknown function also showed altered PSI gene expression ${ }^{20,22}$. This genetic interaction was later substantiated by demonstrating that stand-alone plant SPX proteins can interact with PHR orthologs from Arabidopsis and rice $18,19,23$. The biochemical characterization of SPX domains as cellular receptors for PP-InsPs and the genetic identification of VIH kinases as master regulators of PSR in plants suggested that PP-InsPs, and specifically $\mathrm{Ins}_{8}$ mediates the interaction of SPX proteins with PHRs in response to changing nutrient conditions $\mathrm{s}^{29,30,35}$.

Our quantitative DNA-binding assays demonstrate that AtPHR1 MYB-CC binds the P1BS from the AtSPX1 promoter with high affinity $\left(K_{\mathrm{d}} \sim 0.2 \mu \mathrm{M}\right)$, in agreement with previously reported binding constants for different MYB-CC constructs $\left(K_{\mathrm{d}} \sim 0.01-0.1 \mu \mathrm{M}\right)^{26,39}$. Different oligomeric states have been reported for various PHR MYB-CC constructs ${ }^{2,26}$. Our AtPHR1 MYB-CC construct behaves as a dimer in solution, consistent with the recently reported crystal structure of the AtPHR1 MYB-DNA complex and with earlier reports ${ }^{2,39}$. Purified fulllength OsPHR2, however, appears to be a homotetramer in solution (Supplementary Fig. 3).

In yeast two-hybrid assays, we found that AtSPX1-4 all are able to interact with AtPHR1 (Fig. 1a and Supplementary Fig. 1a). We mapped their conserved interaction surface to the plantunique CC motif of PHRs (Fig. 1a). Crystal structures of this fragment reveal an unusual, four-stranded anti-parallel coiled-coil domain (Fig. 1c). Given the fact, that AtPHR1 MYB-CC forms dimers in solution (Fig. 2b), we cannot exclude the possibility that the CC tetramers represent crystal packing artifacts. However, we did observe identical CC tetramers in three independent crystal lattices (Supplementary Fig. 2) and in solution (Fig. 2a). The residues contributing to the dimer and to the tetramer interfaces are highly conserved among all plant MYB-CC transcription factors (Supplementary Fig. 4). Mutation of either interface blocks AtPHR1 oligomerization in vitro (Fig. 2a, b) and reduces DNA binding (Fig. 2d-f). An attractive hypothesis would thus be that AtPHR1 binds its target promoter as a dimer, but can potentially form homo-tetramers, or hetero-tetramers with other MYB-CC type transcription factors sharing the conserved, plant-unique CC structure and sequence (Supplementary Fig. 4). Notably, PHR1 PHL1 heteromers have been previously described ${ }^{7}$.

We found that SPX-PHR complex formation is mediated by endogenous PP-InsPs in yeast cells, as deletion of the yeast PPInsP kinases Vip1 and Kcs1 abolished the interaction, and mutation of the PP-InsP-binding surface in AtSPX1 interfered with AtPHR1 binding (Supplementary Fig. 1b, c). In line with this, SPX-PHR complexes are found dissociated in vih1 vih2 mutant plants ${ }^{29}$. It is of note that the observed differences in binding affinity for $\mathrm{InsP}_{7}$ and $\mathrm{InsP}_{8}$ to $\mathrm{SPX}-\mathrm{PHR}$ in vitro (Fig. 1b) ${ }^{29}$ cannot fully rationalize the apparent preference for InsP $_{8}$ in vivo $^{29,35}$. We identified the binding surface for SPX-InsP $\mathrm{P}_{8}$ by locating a set of highly conserved basic residues exposed at the surface of the CC domain (Fig. 3a and Supplementary Fig. 4). Mutation of this KHR motif did not strongly impact the ability of isolated OsPHR2 to bind pOsIPS1 in vitro (Fig. 3d), but disrupted the interaction of AtPHR1 with AtSPX1 in yeast (Fig. 3b). The corresponding mutations in OsPHR2 had a similar effect on the interaction with OsSPX4 in quantitative ITC assays (Fig. 3c). Expression of AtPHR $1^{\mathrm{KHR} / \mathrm{A}}$ in the phr1-3 mutant resulted in Pi hyperaccumlation phenotypes and constitutive PSI gene expression in Arabidopsis (Fig. 4a-c). The intermediate growth phenotypes of vih1 vih2 phrl phl1 mutants complemented with AtPHR 1 KHR/A clearly suggests that PP-InsPs do not only regulate the activity of PHR1 and PHL1 in plants, but likely the function of other (SPX domain-containing) proteins $s^{30}$ (Fig. 4e). Notably, binding of AtPHR1 KHR/A to AtSPX1 was reduced in co-immunoprecipitation assays when compared to wild-type AtSPX1 (Fig. 4d and Supplementary Fig. 9). Thus, our and previous finding suggest that $\mathrm{InsP}_{8}$ can promote the association of SPX receptors and PHR transcription factors. The newly identified basic surface area in PHR CC, harboring the conserved KHR motif, likely forms part of the SPX-PHR complex interface (Fig. 3a). 
a

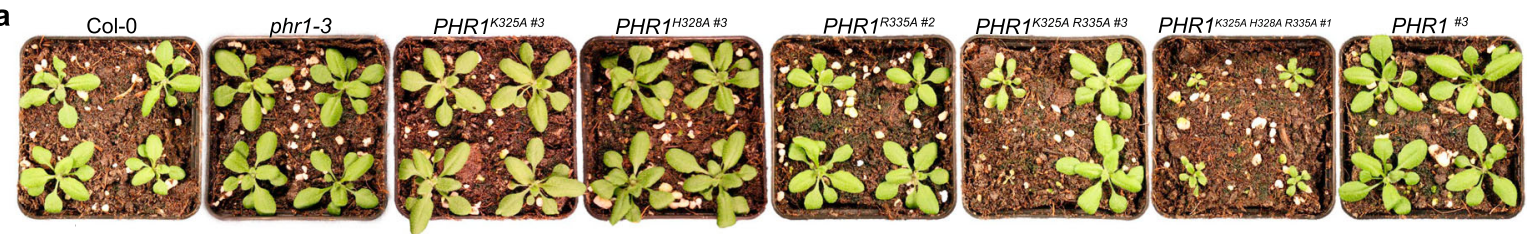

b

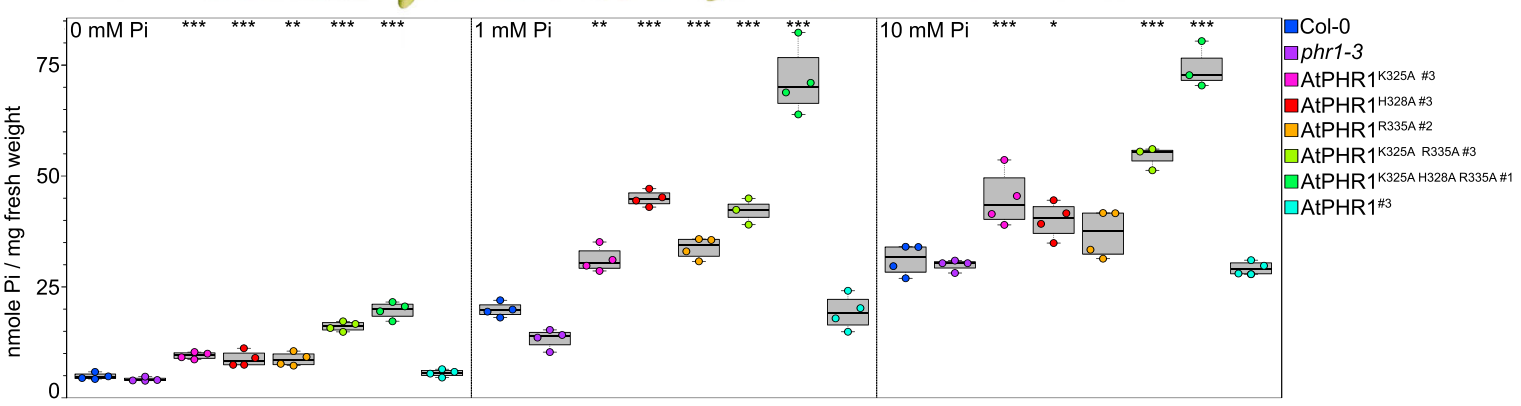

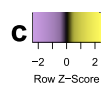

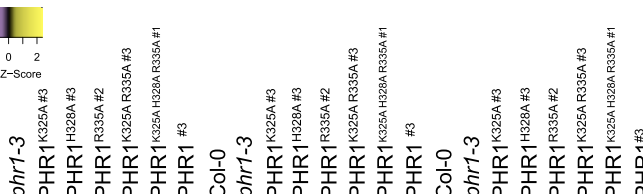

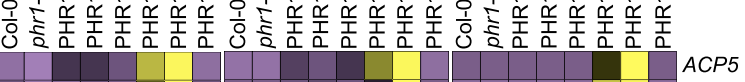
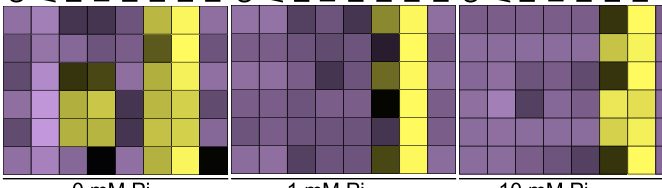

IPS1

$M G D 3$

PECP1

$0 \mathrm{mM} \mathrm{Pi}$

PPsPase

$10 \mathrm{mM} \mathrm{Pi}$

SPX1

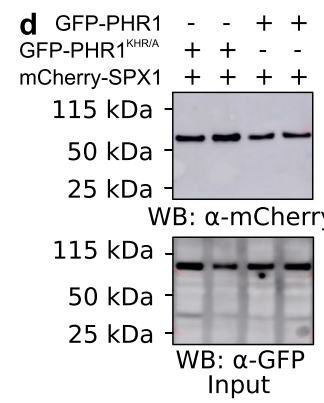

$\underset{\text { GFP-PHR1 }}{\operatorname{maP}-\mathrm{PHR} 1^{\text {KHR/A }}++}+$

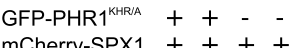

$115 \mathrm{kDa}-$

e

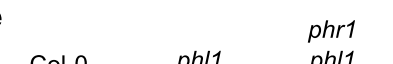
vih1-2 phr1 phl1
phr1 phl1 + eGFP-PHR1
phr1 phl1 + eGFP-PHR1 ${ }^{\text {KHR/A }}$

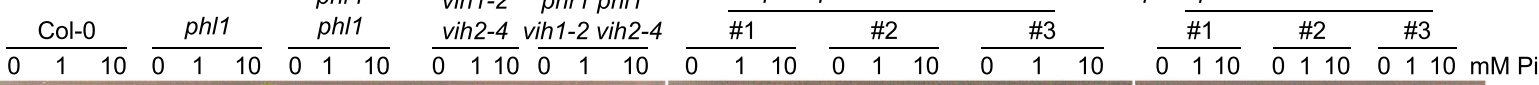

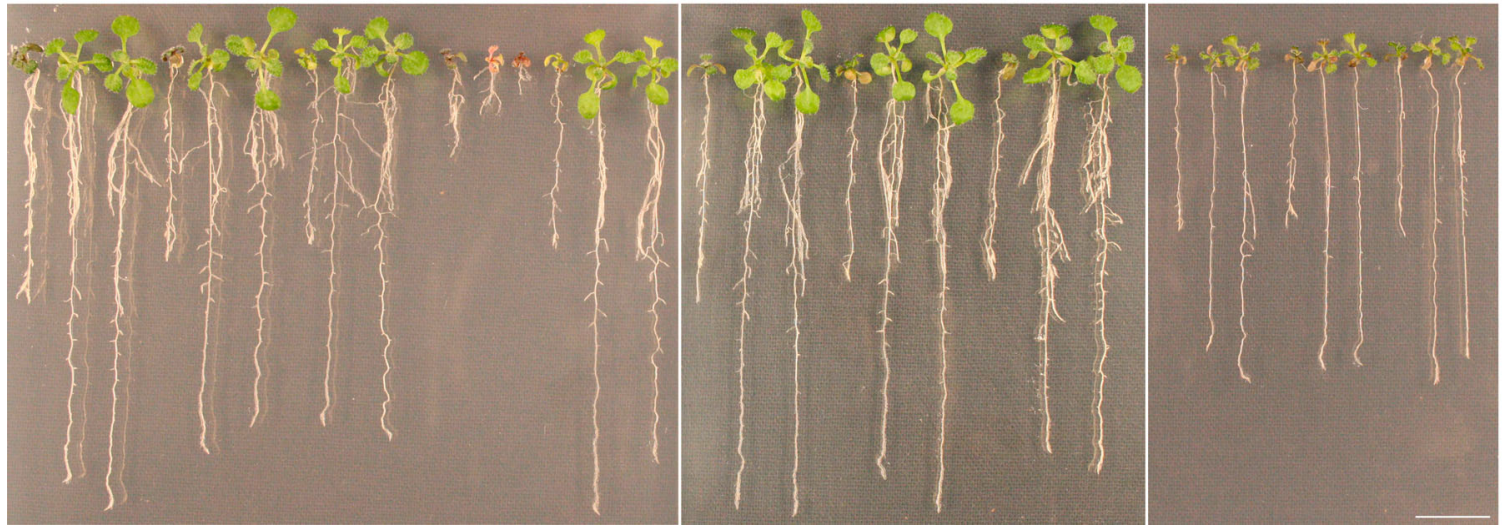

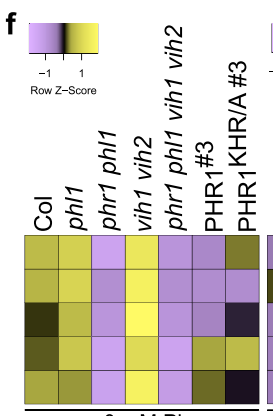

$0 \mathrm{mM} \mathrm{Pi}$

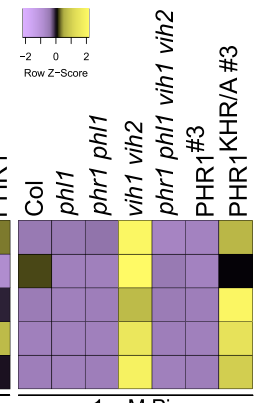

$1 \mathrm{mM} \mathrm{Pi}$

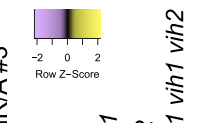

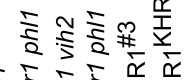

IPS1

$M G D 3$

PECP1

PPSPas

It is of note that addition of AtSPX1 to AtPHR1 has been previously demonstrated to reduce AtPHR1's ability to bind to P1BS in the presence of InsP ${ }_{6}{ }^{26}$. We could not quantify these interactions in ITC or GCI binding assays, as PHR CC formation is much preferred over SPX-Ins $\mathrm{P}_{8}$ binding at the protein concentrations required in these assays. We speculate that $\mathrm{InsP}_{8}$ bound SPX proteins can bind to the basic residues we identified in the PHR CC domain to control the oligomeric state and hence the promoter binding capacity of PHRs. As these residues are conserved among all plant MYB-CC proteins this may suggest that transcription factors outside the PHR subfamily may be regulated by SPX domains and PP-InsPs, possibly rationalizing 
Fig. 4 Mutation of the AtPHR1 KHR motif impairs interaction with AtSPX1 and Pi homeostasis in planta. a Growth phenotypes of Col- 0 wild type, phr1-3

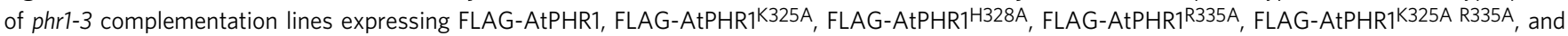
FLAG-AtPHR1 K325A H328A R335A under the control of the AtPHR1 promoter at 21 DAG grown in Pi-sufficient conditions. One representative line per complementation construct is shown (specified by a \#), additional lines are shown in Supplementary Fig. 4. b Plot representing the cellular Pi content of the lines shown in a. Bold black line, median; box, interquartile range (IQR); whiskers, lowest/highest data point within $1.5 \mathrm{IQR}$ of the lower/upper quartile. Seedlings were germinated and grown on vertical ${ }^{1 / 2} \mathrm{MS}$ plates for 8 days, transferred to ${ }^{1 / 2} \mathrm{MS}$ plates supplemented with either 0 , 1 , or $10 \mathrm{mM}$ Pi and grown for additional 7 days. For each line, four plants were measured in technical duplicates. Pi contents of all lines can be found in Supplementary Fig. 4 $\left({ }^{\star} p<0.05 ;{ }^{\star \star} p<0.01 ;{ }^{\star \star \star} p<0.001\right)$. c Heat maps of PSI marker gene (ACP5, IPS1, MGD3, PECP1, PPSPase, SPX1) expression analyses of the lines shown in a, represented as Z-scores. For each line, three biological replicates were analyzed in technical triplicates by qRT-PCR. d Co-immunoprecipitation (Co-IP) experiment assessing the ability for immobilized GFP-AtPHR1 and GFP-AtPHR1KHR/A to interact with mCherry-AtSPX1 in N. benthamiana. Input western blottings are shown alongside. Experiment was performed twice with similar results. e Genetic interactions in the VIH-PHR signaling pathway. Col-O wild type and the indicated mutant seedlings were grown on ${ }^{1 / 2} \mathrm{MS}$ plates for $7 \mathrm{DAG}$, transferred to ${ }^{1 / 2} \mathrm{MS}$ plates supplemented with either 0,1 , or 10 mM $\mathrm{Pi}$ and grown for additional 7 days. For complementation analyses, wild-type AtPHR1 or AtPHR1KHR/A was expressed as an N-terminal eGFP fusion protein under the control of the AtPHR1 promoter. $\mathbf{f}$ Heat maps of PSI marker gene expression for the lines shown in $\mathbf{e}$.

the severe phenotypes of vih1 vih2 mutant plants (Fig. 4e and Supplementary Fig. 4). The recent findings that VIH kinases and PHRs act together in plant PSR and that SPX-PHR complexes are dissociated in vih1 vih2 mutants further suggest that $\mathrm{InsP}_{8}$ is the bona fide signaling molecule promoting the association between SPX domains and MYB-CCs ${ }^{29,35}$. Repressive SPX-PHR complexes consequently form only under Pi-sufficient conditions, where $\mathrm{InsP}_{8}$ levels are high ${ }^{29,35}$. Under Pi starvation, when $\mathrm{InsP}_{8}$ levels are reduced, SPX-PHR complexes dissociate, enabling the transcription factors to acquire the oligomeric state required for high affinity promoter binding. The physiological and mechanistic investigation of this central process may, in the long term, contribute to the development of $\mathrm{Pi}$ starvation resilient crops. This could in turn sustain the use of the essential and nonrenewable resource rock phosphate, which is currently consumed at an alarming scale.

\section{Methods}

Molecular cloning, constructs, and primers. For a detailed description of the cloning strategies, constructs and primers used in this study, please refer to Supplementary Tables 2 and 3 .

Generation of stable transgenic A. thaliana lines. All stable transgenic $A$. thaliana lines are listed in Supplementary Table 1. Constructs were introduced into Agrobacterium tumefaciens strain pGV2260 and A. thaliana plants were transformed via floral dipping ${ }^{46}$. Transformants were identified by mCherry fluorescence with a Zeiss Axio Zoom.V16 stereo microscope (mRFP filter) and a HXP200C illuminator. Homozygous T3 lines have been identified for complementation lines expressing FLAG-AtPHR1, FLAG-AtPHR1 K325A, FLAGAtPHR 1 H328A, and FLAG-AtPHR1 ${ }^{\mathrm{R} 335 \mathrm{~A}}$ under the control of the native AtPHR1 promoter. For complementation lines expressing FLAG-AtPHR1 K325A R335A and FLAG-AtPHR 1 K325A H328A R335A under the control of the native AtPHR1 promoter, T2 lines were used throughout, and homozygous and heterozygous transformants were selected for all experiments by mCherry fluorescence as described above. T3 homozygous lines expressing eGFP-PHR1 under the control of the AtPHR1 promoter were identified by their Hygromycin resistance. PHR1 was amplified from Arabidopsis cDNA and introduced into $\mathrm{pH} 7 \mathrm{~m} 34 \mathrm{GW}$ binary vector. Point mutations were introduced by site-directed mutagenesis (primers are listed in Supplementary Table 3).

Yeast two-hybrid experiments. (Screen) AtSPX1 ${ }^{1-252}$ was used as a bait and screened against an A. thaliana seedling cDNA library by Hybrigenics Services. AtSPX1 $1^{1-252}$ was cloned into the pB29 vector providing a C-terminal LexA-DNAbinding domain and transformed into yeast strain L40aGal4 (MATa). Prey genes were cloned into the pP6 vector providing a N-terminal Gal4-activation domain and transformed into yeast strain YHGX13 (MATa). After mating haploid bait and prey strains, positive interactions were detected by growth on histidine deficient medium.

Yeast strains and media. For all experiments, either the diploid TATA strain (Hybrigenics Services) or the haploid L40 strain was used (Supplementary Table 2). Cells were routinely maintained on yeast extract-peptone-adenine-dextrose (YPAD) plates $(20 \mathrm{~g} / \mathrm{L}$ glucose, $20 \mathrm{~g} / \mathrm{L}$ bacto-peptone, $10 \mathrm{~g} / \mathrm{L}$ yeast extract, $0.04 \mathrm{~g} / \mathrm{L}$ adenine hemisulfate, and $20 \mathrm{~g} / \mathrm{L}$ agar). Experiments were performed on synthetic dropout $(\mathrm{SD})$ plates $(6.7 \mathrm{~g} / \mathrm{L}$ yeast nitrogen base with adenine hemisulfate and without leucine, tryptophan, and histidine, $20 \mathrm{~g} / \mathrm{L}$ glucose, and $20 \mathrm{~g} / \mathrm{L}$ agar) supplemented with $0.076 \mathrm{~g} / \mathrm{L}$ histidine or $10 \mathrm{mM} \mathrm{3-amino,1,2,4-triazole} \mathrm{(3-AT)}$

Yeast transformation. One yeast colony was resuspended in $500 \mu \mathrm{L}$ sterile $\mathrm{H}_{2} \mathrm{O}$ plated on YPAD plates, and grown for 2 days until the whole plate was covered with yeast. Yeast cells were then resuspended in $50 \mathrm{~mL}$ YPAD liquid medium and the $\mathrm{OD}_{600 \mathrm{~nm}}$ was determined $\left(2 \times 10^{6}\right.$ cells $/ \mathrm{mL}$ were used for one transformations). Cells were centrifuged at $3000 \times g$ and $4{ }^{\circ} \mathrm{C}$ for $5 \mathrm{~min}$, resuspended in $25 \mathrm{~mL}$ TrisEDTA (TE) buffer, centrifuged again at $3000 \times g$ and $4^{\circ} \mathrm{C}$ for $5 \mathrm{~min}$, resuspended in $2 \mathrm{~mL}$ LiAc/TE buffer, centrifuged at $16,000 \times g$ and room temperature (RT) for $15 \mathrm{~s}$, and finally resuspended in $50 \mu \mathrm{L} /$ transformation LiAc/TE buffer. The transformation mix $(0.5 \mu \mathrm{g}$ bait plasmid, $0.5 \mu \mathrm{g}$ prey plasmid, $10 \mu \mathrm{L} s \mathrm{sDNA}(10 \mathrm{mg} / \mathrm{mL})$, $50 \mu \mathrm{L}$ yeast cells, $345 \mu \mathrm{L} 40 \%$ (w/v) PEG3350 in LiAC/TE) was prepared and incubated at $30^{\circ} \mathrm{C}$ for $45 \mathrm{~min}$, followed by incubation at $42{ }^{\circ} \mathrm{C}$ for $30 \mathrm{~min}$. Finally, yeast cells were centrifuged at $6500 \times g$ and RT for $15 \mathrm{~s}$, resuspended in TE buffer, plated on SD plates lacking leucine and tryptophan, and incubated at $30^{\circ} \mathrm{C}$ for 3 days.

Yeast spotting dilution assay. Positive transformants were selected on SD plates without tryptophan and leucine, and incubated at $30{ }^{\circ} \mathrm{C}$ for three days. Cells were counted, washed in sterile water and spotted in 5 times dilution $(5000,1000,200$, 40 , and 8 cells) on SD plates without either tryptophan and leucine, or tryptophan, leucine, and histidine supplemented with $10 \mathrm{mM} \mathrm{3-AT}$. Plates were incubated at $30{ }^{\circ} \mathrm{C}$ for 3 days.

Protein expression and purification. For in vitro biochemistry, AtPHR1 $280-360 \mathrm{wt} /$ KHR/Olig1/Olig2 and AtPHR1 222-358 wt/Olig1/Olig2 were cloned into the pMH-HT protein expression vector, providing a $\mathrm{N}$-terminal $6 \times$ His affinity tag with a tobacco etch virus (TEV) protease recognition site. OsPHR2 ${ }^{1-426 \mathrm{wt} / \mathrm{KHR} / \mathrm{A}}$ was cloned into the $\mathrm{pMH}-\mathrm{HSgb} 1 \mathrm{~T}$ protein expression vector, providing a $\mathrm{N}$-terminal $8 \times$ His-StrepGB1 affinity tag with a TEV cleavage site. OsSPX2 ${ }^{1-321}$ was cloned into the pMHHSsumo protein expression vector, providing a N-terminal 8xHis-Strep-Sumo affinity tag. All constructs were transformed into Escherichia coli BL21 (DE3) (argU, ileY, leuW) RIL cells. For recombinant protein expression, cells were grown at $37^{\circ} \mathrm{C}$ in terrific broth (TB) medium to an $\mathrm{OD}_{600 \mathrm{~nm}}$ of $\sim 0.6$. After reducing the temperature to $18{ }^{\circ} \mathrm{C}$, protein expression was induced with $0.3 \mathrm{mM}$ isopropyl $\beta$-D-galactoside (IPTG) for $16 \mathrm{~h}$. Cells were centrifuged at $4500 \times g$ and $4{ }^{\circ} \mathrm{C}$ for $1 \mathrm{~h}$, resuspended in lysis buffer $(50 \mathrm{mM}$ Tris- $\mathrm{HCl} \mathrm{pH} 7.8,500 \mathrm{mM} \mathrm{NaCl}, 0.1 \%$ (v/v) IGEPAL, $1 \mathrm{mM}$ $\mathrm{MgCl}, 2 \mathrm{mM} \beta$-mercaptoethanol), snap-frozen in liquid nitrogen, and stored at $-80^{\circ} \mathrm{C}$. For protein preparation, cells were thawed, supplemented with cOmplete ${ }^{\mathrm{TM}}$ EDTA-free protease inhibitor cocktail (Roch), DNaseI, and lysozyme, and disrupted using a sonicator. Cell lysates were centrifuged at $7000 \times g$ and $4{ }^{\circ} \mathrm{C}$ for $1 \mathrm{~h}$, sterile filtered, supplemented with $20 \mathrm{mM}$ imidazole, and loaded onto a $5 \mathrm{~mL}$ HisTrap HP $\mathrm{Ni}^{2+}$ affinity column (GE Healthcare). After washing with several column volumes (CVs) of lysis buffer supplemented with $20 \mathrm{mM}$ imidazole, high-salt buffer (50 mM Tris- $\mathrm{HCl} \mathrm{pH} 7.8,1 \mathrm{M} \mathrm{NaCl}, 2 \mathrm{mM} \beta$-mercaptoethanol), and high-phosphate buffer (200 $\mathrm{mM} \mathrm{K}_{2} \mathrm{HPO}_{4} / \mathrm{KH}_{2} \mathrm{PO}_{4} \mathrm{pH} 7.8,2 \mathrm{mM} \beta$-mercaptoethanol), proteins were eluted in a gradient from 20 to $500 \mathrm{mM}$ imidazole in lysis buffer. The purified proteins were cleaved by TEV or Sumo protease overnight at $4{ }^{\circ} \mathrm{C}(1: 100$ ratio) and dialyzed against lysis buffer for PHR1 222-358 wt/Olig1/Olig2 and PHR1 280-360 wt/KHR/Olig1/Olig2 fragments, against modified lysis buffer $(25 \mathrm{mM}$ Tris- $\mathrm{HCl} \mathrm{pH} 7.8,300 \mathrm{mM}$ $\mathrm{NaCl}, 0.1 \%$ (v/v) IGEPAL, $1 \mathrm{mM} \mathrm{MgCl}, 2 \mathrm{mM} \beta$-mercaptoethanol) for OsPHR2 ${ }^{1-426} \mathrm{wt} / \mathrm{KHR} / \mathrm{A}$, and against modified anion exchange buffer (20 mM Tris$\mathrm{HCl} \mathrm{pH} \mathrm{6.5,500} \mathrm{mM} \mathrm{NaCl}$ ) for OsSPX4 ${ }^{1-321}$. PHR1 $280-360 \mathrm{wt} / \mathrm{KHR} / \mathrm{Olig} 1 / \mathrm{Olig} 2$ and OsPHR2 $2^{1-426 \mathrm{wt} / \mathrm{KHR} / \mathrm{A}}$ were subjected to a second $\mathrm{Ni}^{2+}$ affinity purification in either lysis buffer or modified lysis buffer, respectively, and the flow-throughs were collected and concentrated. PHR1 $222-358 \mathrm{wt} / \mathrm{Olig} 1 / \mathrm{Olig} 2$ were subjected to cation exchange (50 $\mathrm{mM}$ HEPES pH 7.5, 50-1000 $\mathrm{mM} \mathrm{NaCl}$ ) and OsSPX $4^{1-321}$ was subjected to anion exchange $(20 \mathrm{mM}$ Tris- $\mathrm{HCl}, 50-1000 \mathrm{mM} \mathrm{NaCl})$. Fractions corresponding to 
the respective proteins were pooled and concentrated. All proteins were loaded onto a HiLoad Superdex $75 \mathrm{pg}$ HR26/60 column (GE Healthcare), pre-equilibrated in gel filtration buffer A (20 mM Tris/ $\mathrm{HCl} \mathrm{pH} \mathrm{7.5,} 300 \mathrm{mM} \mathrm{NaCl}, 0.5 \mathrm{mM}$ tris(2-carboxyethyl)phosphine (TCEP)) for PHR1 222-358 wt/olig1/Olig2, or in gel filtration buffer B (20 mM Tris/HCl pH 7, $200 \mathrm{mM} \mathrm{NaCl}, 0.5 \mathrm{mM} \mathrm{TCEP}$ ) for the remaining proteins. Fractions containing the respective proteins were pooled and concentrated. Purified and concentrated protein was immediately used for further experiments or snapfrozen in liquid nitrogen and stored at $-80^{\circ} \mathrm{C}$

The AtPHR $1^{280-360}$ fragment used for crystallization was cloned into the $\mathrm{pMH}$ HS-Sumo protein expression vector, providing a $\mathrm{N}$-terminal $8 \times$ His-StrepII tandem affinity tag and a Sumo fusion protein. The construct was transformed into E. coli BL21 (DE3) RIL cells. For recombinant protein expression, cells were grown at $37^{\circ} \mathrm{C}$ in $\mathrm{TB}$ medium to an $\mathrm{OD}_{600 \mathrm{~mm}}$ of $\sim 0.6$. After reducing the temperature to $16^{\circ} \mathrm{C}$, protein expression was induced with $0.3 \mathrm{mM}$ IPTG for $16 \mathrm{~h}$. Cells were centrifuged for $20 \mathrm{~min}$ at $4000 \times \mathrm{g}$ and $4^{\circ} \mathrm{C}$, then the cell pellet was washed with PBS, snap-frozen in liquid nitrogen and stored at $-80^{\circ} \mathrm{C}$. For the purification of a putative AtSPX1-InsP $\mathrm{P}_{8}$-AtPHR1 ${ }^{280-360}$ complex, the AtPHR1 cell pellet was thawed and mixed with twice the amount of cells expressing a His-Strep-MBPAtSPX1 $1^{1-251}$ fusion protein, which provides a N-terminal, TEV-cleavable MBP. Lysis buffer (200 mM KP $\mathrm{i}$ pH 7.8, $2 \mathrm{mM} \beta$-mercaptoethanol) supplemented with $0.1 \%$ (v/v) IGEPAL, $1 \mathrm{mM} \mathrm{MgCl} 2,10 \mathrm{mM}$ imidazole, 500 units TurboNuclease

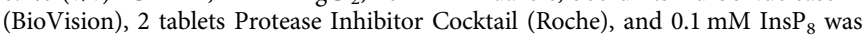
added and cells were disrupted using an EmulsiFlex-C3 (Avestin). Cell lysates were centrifuged at $7000 \times g$ and $4{ }^{\circ} \mathrm{C}$ for $1 \mathrm{~h}$. The cleared supernatant was sterile filtered and loaded onto a $5 \mathrm{~mL}$ HisTrap HP Ni ${ }^{2+}$ affinity column (GE Healthcare). After washing with several CVs of lysis buffer, the protein was eluted with $250 \mathrm{mM}$ imidazole in lysis buffer. The purified His-Strep-Sumo-AtPHR1/His-Strep-MBPAtSPX1 fusion proteins were cleaved by TEV and Sumo protease treatment overnight at $4{ }^{\circ} \mathrm{C}$, while dialyzing in a buffer containing $200 \mathrm{mM} \mathrm{KP}$ i pH 7.8, 100 $\mathrm{mM} \mathrm{NaCl}, 2 \mathrm{mM} \beta$-mercaptoethanol. Imidazole $(10 \mathrm{mM})$ was added to the cleaved protein sample and a second $\mathrm{Ni}^{2+}$ affinity step was performed in order to remove the cleaved-off His-Strep-Sumo/MBP fusion tags as well as the $6 \times$ His-tagged Sumo and TEV proteases. The flow-through was concentrated and loaded onto a HiLoad Superdex $75 \mathrm{pg}$ HR16/60 column (GE Healthcare), pre-equilibrated in gel filtration buffer $\left(20 \mathrm{mM}\right.$ Tris/ $\mathrm{HCl} \mathrm{pH} \mathrm{7.8,} 250 \mathrm{mM} \mathrm{NaCl}, 2.5 \mathrm{mM} \mathrm{InsP}_{6}, 0.5 \mathrm{mM}$ TCEP). Fractions containing the co-eluting AtSPX1 $1^{1-251}$ and AtPHR1 ${ }^{280-360}$ proteins were pooled and concentrated. A second SEC step was performed using a HiLoad Superdex $200 \mathrm{pg}$ HR26/60 column (GE Healthcare) and the same gel filtration buffer as above. It was later found that AtSPX1 ${ }^{1-251}$ expressed in $E$. coli is largely unfolded and unable to bind InsP $\mathrm{P}_{8}$ and hence no complex with AtPHR $1^{280-360}$ was observed in our crystals. Purified and concentrated protein was immediately used for further experiments or snap-frozen in liquid nitrogen and stored at $-80^{\circ} \mathrm{C}$.

For production of $\left[\mathrm{U}-{ }^{15} \mathrm{~N},{ }^{2} \mathrm{H}\right]$-labeled AtPHR $1^{280-360}$ variants for NMR spectroscopy, AtPHR1 ${ }^{280-360}$ and AtPHR1 $1^{280-360 ~ K H R / A}$ in PMH-HT were transformed and expressed in E. coli BL21 (DE3) RIL cells. Luria-Beltani liquid medium $(2 \mathrm{~mL})$ was inoculated with freshly transformed cells. After overnight growth at $37^{\circ} \mathrm{C}$, cells were transferred to $\mathrm{M} 9_{\mathrm{H} 2 \mathrm{O}}$ medium and grown overnight at $37^{\circ} \mathrm{C}$. From this culture, $150 \mu \mathrm{L}$ were transferred into $1 \mathrm{~mL} \mathrm{M} 9_{\mathrm{H} 2 \mathrm{O}}$ medium and grown at $37^{\circ} \mathrm{C}$ overnight. Cells were transferred into $100 \mathrm{~mL} \mathrm{M9} 9_{\mathrm{D} 2 \mathrm{O}}$ medium and grown overnight at $37^{\circ} \mathrm{C}$. The culture was added to $0.9 \mathrm{~L}$ of $\mathrm{M} 9_{\mathrm{D} 2 \mathrm{O}}$ medium and grown at $37^{\circ} \mathrm{C}$ to an $\mathrm{OD}_{600 \mathrm{~nm}}$ of $\sim 0.4$, then the temperature was shifted to $16^{\circ} \mathrm{C}$. After $1 \mathrm{~h}$, protein expression was induced with $0.3 \mathrm{mM}$ IPTG and cells were grown overnight, reaching a final $\mathrm{OD}_{600 \mathrm{~nm}}$ of 1.2-1.4. The cell culture was harvested at $7800 \times g$ and the cell pellet was stored at $-20^{\circ} \mathrm{C}$. The cell pellet was resuspended in $50 \mathrm{mM}$ Tris pH 7.8, $500 \mathrm{mM} \mathrm{NaCl}, 0.1 \%$ IGEPAL, $1 \mathrm{mM} \mathrm{MgCl} 2,2 \mathrm{mM} \beta$ mercaptoethanol, $20 \mathrm{mM}$ imidazole, and DNAseI (AppliChem). The resuspension was homogenized by magnetic stirring and after $10 \mathrm{~min}$ lysozyme was added. The cells were lysed by sonication and subsequently centrifuged at $42,500 \times g$ for 45 min. The collected supernatant was applied on $5 \mathrm{~mL}$ Histrap HP (GE Healthcare) equilibrated in a buffer A ( $50 \mathrm{mM}$ Tris $\mathrm{pH} 7.8,500 \mathrm{mM} \mathrm{NaCl}$ and $2 \mathrm{mM} \beta$ mercaptoethanol) and washed with a wash buffer $1(50 \mathrm{mM}$ Tris $\mathrm{pH} 7.8,200 \mathrm{mM}$ $\mathrm{KPi}$, and $2 \mathrm{mM} \beta$-mercaptoethanol) and with wash buffer $2(50 \mathrm{mM}$ Tris $\mathrm{pH} 7.8$, $1 \mathrm{M} \mathrm{NaCl}, 2 \mathrm{mM} \beta$-mercaptoethanol), followed by a final wash step with buffer A. His-tagged protein was eluted with a buffer B $(50 \mathrm{mM}$ Tris pH 7.8, $500 \mathrm{mM} \mathrm{NaCl}$, $1 \mathrm{M}$ imidazole, and $1 \mathrm{mM} \beta$-mercaptoethanol). The fractions containing the eluted protein were incubated with TEV protease overnight at $4{ }^{\circ} \mathrm{C}$. The sample was dialyzed against buffer $\mathrm{A}$ at $4{ }^{\circ} \mathrm{C}$ and purified by a second $\mathrm{Ni}^{2+}$ affinity step. Cleaved AtPHR1 ${ }^{280-360}$ from the flow-through of the column was concentrated to $3 \mathrm{~mL}$ and purified further by SEC on a Superdex 75 16/600 (GE Healthcare) equilibrated in $25 \mathrm{mM}$ HEPES pH 7.0, $200 \mathrm{mM} \mathrm{NaCl}$ and $0.5 \mathrm{mM}$ TCEP at a flow rate of $1 \mathrm{~mL} / \mathrm{min}$. Fractions containing AtPHR $1^{280-360}$ were pooled, concentrated to $0.5 \mathrm{~mL}$, and the protein concentration was determined via Bradford assay (AppliChem). Samples were flash-frozen in liquid nitrogen and subsequently used for NMR spectroscopy experiments.

Isothermal titration calorimetry. All ITC experiments were performed at $25^{\circ} \mathrm{C}$ using a MicroCal PEAQ-ITC system (Malvern Panalytical) equipped with a $200 \mu \mathrm{l}$ sample cell and a $40 \mu$ injection syringe. $\mathrm{Ins}_{7}$ and $\mathrm{Ins}_{8}$ were produced as described ${ }^{47}$. All proteins were dialyzed against ITC buffer (20 mM HEPES pH 7.0,
$200 \mathrm{mM} \mathrm{NaCl}$ ) and PP-InsP ligands were diluted in ITC buffer prior to all measurements. A typical titration consisted of 15 injections, the protein concentrations in the syringe and in the cell are provided in the respective figure legend. Data were analyzed using the MicroCal PEAQ-ITC analysis software (v1.21).

Crystallization and crystallographic data collection. Two hexagonal crystal forms containing AtPHR $1^{280-360}$ only developed in sitting drops consisting of $0.2 \mu \mathrm{L}$ protein at a concentration of $12 \mathrm{mg} / \mathrm{mL}$ and $0.2 \mu \mathrm{L}$ reservoir solution $(0.1 \mathrm{M}$ phosphate citrate $\mathrm{pH} 4.2,0.2 \mathrm{M} \mathrm{NaCl}, 20 \%$ (w/v) PEG 8000). Crystals were cryoprotected by adding reservoir solution containing $10 \%(\mathrm{v} / \mathrm{v})$ ethylene glycol directly to the drop and subsequently snap-frozen in liquid nitrogen. A third, tetragonal crystal form developed in $0.1 \mathrm{M}$ Bis-Tri pH 6.5, $0.1 \mathrm{M} \mathrm{NaCl}, 1.5 \mathrm{M}\left(\mathrm{NH}_{4}\right)_{2} \mathrm{SO}_{4}$. Crystals were cryo-protected by serial transfer into reservoir solution supplemented with $15 \%(\mathrm{v} / \mathrm{v})$ glycerol and snap-frozen in liquid nitrogen. Crystal forms 1, 2, and 3 diffracted to $\sim 2.4, \sim 2.5$, and $\sim 1.9 \AA$ resolution, respectively. Data were collected at beam-line PXIII of the Swiss Light Source, Villigen, Switzerland. Data processing and scaling were done in $\mathrm{XDS}^{48}$.

Crystallographic structure solution and refinement. The AtPHR1 $1^{280-360}$ structure was solved by molecular replacement using the previously described SPX CtGdel (PDB-ID:5IJJ) core helices as search model in calculations with the program PHASER ${ }^{49}$. The structure was completed in iterative cycles of manual model building in COOT $^{50}$ and restrained refinement in phenix.refine ${ }^{51}$ or Refmac5 ${ }^{52}$. Residues $280-294$ and 278-280 appear disordered in the final model. Quality of the structural model was assessed by using MolProbity ${ }^{53}$, refinement statistics are shown in Supplementary Table 5. Structure visualization was done with PyMOL (Molecular Graphics System, Version 1.8, Schrödinger, LLC) and ChimeraX ${ }^{54}$. The structure of AtSPX1 was modeled using the program SWISSMODEL $^{55}$ and the SPX ${ }^{\mathrm{HsXPR} 1}$ domain structure of the human phosphate exporter as template (PDB-ID:5IJH, GMQE score 0.49, QMEAN4 score -2.27, 29.5\% sequence identity $\left.{ }^{30}\right)$. Conserved PP-InsP-binding residues in AtSPX1 were determined by aligning sequences with previously described SPX domains ${ }^{30}$ using the program T-coffee ${ }^{56}$.

Right-angle light scattering. The oligomeric state of AtPHR1 variants was analyzed by SEC paired with a refractive index detector using an OMNISEC RESOLVE/REVEAL combined system (Malvern). Instrument calibration was performed with a bovine serum albumin (BSA) standard (Thermo Scientific Albumin Standard). Samples of $50 \mu \mathrm{L}$ containing $2-10 \mathrm{mg} / \mathrm{mL}$ AtPHR1 (wild-type AtPHR1280-360, AtPHR1 280-360 Olig1, AtPHR1 280-360 Olig2, AtPHR1280-360 KHR/A, wild-type AtPHR1 222-358, AtPHR1 122-358 Olig1, and AtPHR1222-358 Olig2) in OMNISEC buffer (20 mM HEPES pH 7.5, $150 \mathrm{mM} \mathrm{NaCl}$ ) were separated on a Superdex 200 increase 10/300 GL column (GE Healthcare) at a column temperature of $25^{\circ} \mathrm{C}$ and a flow rate of $0.7 \mathrm{ml} \mathrm{min}{ }^{-1}$. Data were analyzed using the OMNISEC software (v10.41).

DNA oligonucleotide annealing. DNA oligonucleotides were dissolved in annealing buffer (10 mM HEPES- $\mathrm{NaOH} \mathrm{pH} 8.0,50 \mathrm{mM} \mathrm{NaCl}, 0.1 \mathrm{mM}$ EDTA), Equal volumes of the equimolar DNA oligonucleotides were mixed and incubated in a heat block for $5 \mathrm{~min}$ at $95^{\circ} \mathrm{C}$. Subsequently, DNA oligonucleotides were cooled down to room temperature for $90 \mathrm{~min}$. Double-stranded DNA oligonucleotides were aliquoted and stored at $-20^{\circ} \mathrm{C}$

Electrophoretic mobility shift assay. Mini-PROTEAN TBE precast gels (5\%; BioRad) have been pre-electrophoresed in $0.5 \times$ TBE buffer for $60 \mathrm{~min}$ at $70 \mathrm{~V}$. Reactions mixes have been prepared following the Odyssey Infrared EMSA kit manual (LI-COR) without the use of optional components, including $50 \mathrm{nM}$ of IRDye800 end-labeled oligos (refer to Supplementary Table 4a; Metabion), and a $1: 5$ dilution series of wild-type AtPHR1 $1^{222-358}$ or AtPHR $1^{222-358}$ Olig1 $(1.2 \mu \mathrm{g}$ to $76.8 \mathrm{pg}$ ). Reaction mixes have been incubated for $30 \mathrm{~min}$ at room temperature in the dark and $2 \mu \mathrm{L}$ of $10 \times$ Orange Loading Dye (LI-COR) have been added to each sample prior to loading on a 5\% TBE gel. Gels have been electrophoresed until orange dye migrated to the bottom of the gel $(\sim 1 \mathrm{~h})$ at $70 \mathrm{~V}$ in the dark. Gels have been scanned with the $800 \mathrm{~nm}$ channel of an Odyssey imaging system (LI-COR)

Grating-coupled interferometry. All GCI experiments were performed at $4{ }^{\circ} \mathrm{C}$ using a Creoptix WAVE system (Creoptix sensors) with 4PCP WAVE chips (Creoptix sensors). Chips were conditioned with borate buffer (100 mM sodium borate $\mathrm{pH} 9.0,1 \mathrm{M} \mathrm{NaCl}$ ) and subsequently neutravidin was immobilized on the chip surface via standard amine-coupling: activation $(1: 1 \mathrm{mix}$ of $400 \mathrm{mM}$ $N$-(3-dimethylaminopropyl)- $N^{\prime}$-ethylcarbodiimide hydrochloride and $100 \mathrm{mM}$ $N$-hydroxysuccinimide), immobilization ( $30 \mu \mathrm{g} \mathrm{ml}^{-1}$ of neutravidin in $10 \mathrm{mM}$ sodium acetate, $\mathrm{pH} 5.0$ ), passivation (5\% BSA in $10 \mathrm{mM}$ sodium acetate $\mathrm{pH} 5.0$ ), and quenching (1 M ethanolamine, $\mathrm{pH}$ 8.0). Biotinylated oligos (Supplementary Table $4 \mathrm{~b}$; Metabion) were captured on the chip. Analytes were injected in a $1: 2$ dilution series starting from $4 \mu \mathrm{M}$ (AtPHR1 $222-358), 20 \mu \mathrm{M}$ (AtPHR1 $223-358$ Olig1, AtPHR1 223-358 Olig2), or $10 \mu \mathrm{M}$ (OsPHR2, OsPHR2 KHR/A) in GCI buffer (for OsPHR2: $20 \mathrm{mM}$ HEPES pH 7.9, $200 \mathrm{mM} \mathrm{NaCl}$; for AtPHR1: $20 \mathrm{mM}$ HEPES pH 
$7.5,300 \mathrm{mM} \mathrm{NaCl})$. Blank injections every fourth cycle were used for double referencing and a dimethylsulfoxide (DMSO) calibration curve $(0 \%, 0.5 \%, 1 \%$, $1.5 \%, 2 \%)$ for bulk correction. Data were corrected and analyzed using the Creoptix WAVE control software (corrections applied: X and Y offset, DMSO calibration, double referencing, refractive index correction), and a one-to-one binding model was used to fit all experiments.

CD spectroscopy. Far-UV CD spectra and melting curves were acquired with a Chirascan V100 CD spectrometer holding the protein solutions in quartz cuvettes of $1 \mathrm{~mm}$ optical path, scanning from 260 to $190 \mathrm{~nm}$ at $1 \mathrm{~nm} / \mathrm{s}$ with a slit width of $1 \mathrm{~nm}$ against an air background obtained with the same settings without any cuvette. Protein concentration was $0.08 \mathrm{mg} / \mathrm{mL}(8.5 \mu \mathrm{M})$ in all experiments, the buffer was $10 \mathrm{mM} \mathrm{KPi} \mathrm{pH} \mathrm{7.5.} \mathrm{A} \mathrm{reference} \mathrm{spectrum} \mathrm{of} \mathrm{the} \mathrm{buffer} \mathrm{showed} \mathrm{only} \mathrm{noise} \mathrm{within}$ $\pm 0.5 \mathrm{mdeg}$. All the final spectra reported were obtained with a single scan, as we observed very good signal-to-noise and no saturation of the detector voltage even at low wavelengths. Melting curves were acquired with $2{ }^{\circ} \mathrm{C}$ stepwise increments and $30 \mathrm{~s}$ intervals from 24 to $98^{\circ} \mathrm{C}$. Data were fitted in the range from $195 \mathrm{~nm}$ to $250 \mathrm{~nm}$ using the three-component (a-helix, $\beta$-strand, and random coil) fitting (http:// lucianoabriata.altervista.org/jsinscience/cd/cd3.html) as previously described ${ }^{57}$.

NMR spectroscopy. TROSY spectra of $\left[\mathrm{U}-{ }^{15} \mathrm{~N},{ }^{2} \mathrm{H}\right]-\mathrm{AtPHR} 1^{280-360}$ and $\left[\mathrm{U}^{15} \mathrm{~N},{ }^{2} \mathrm{H}\right]-\mathrm{AtPHR}{ }^{1280-360 ~ K H R / A}$ were acquired on a $600 \mathrm{MHz}$ spectrometer with a cryo-probe at $30^{\circ} \mathrm{C}$. The NMR buffer contained $25 \mathrm{mM}$ HEPES pH 7.0, $200 \mathrm{mM}$ $\mathrm{NaCl}$ and $0.5 \mathrm{mM}$ TCEP. NMR data were analyzed by CCPNMR 2.4.258. The dissociation constant $K_{\mathrm{D}}$ was obtained by calculating chemical shift perturbations of each point by and plotted against InsP $\mathrm{P}_{8}$ concentration. The data points were fitted in MATLAB.

Plant material, seed sterilization, and plant growth conditions. All A. thaliana plants used in this study were of the Columbia (Col-0) ecotype. Seeds of the T-DNA insertion lines phr1-3 (SALK_067629) and phl1 (SAIL_731_B09) were obtained from the European Arabidopsis Stock Center. Homozygous phr1-3 and phll lines were identified by PCR using T-DNA left and right border primers paired with gene-specific sense and antisense primers (Supplementary Table 3d). The phrl phl1 double mutant was kindly provided by Yves Poirier (University of Lausanne, Switzerland); vih1-2 vih2-4 double and phr1 phl1 vih1-2 vih2-4 quadruple mutants have been reported previously ${ }^{35}$. Seeds were surface sterilized by incubation in $70 \%(\mathrm{v} / \mathrm{v}) \mathrm{CH}_{3}-\mathrm{CH}_{2}-\mathrm{OH}$ for $10 \mathrm{~min}$, followed by incubation in $0.5 \%$ $(\mathrm{v} / \mathrm{v})$ sodium hypochlorite for $10 \mathrm{~min}$, and subsequently washed four times in sterile $\mathrm{H}_{2} \mathrm{O}$. Seeds were placed on full half-strength Murashige-Skoog plates ${ }^{59}$ containing $1(\mathrm{w} / \mathrm{v}) \%$ sucrose and $0.8(\mathrm{w} / \mathrm{v}) \%$ agar $\left({ }^{1 / 2} \mathrm{MS}\right.$ plates), and stratified for $2-3$ days at $4{ }^{\circ} \mathrm{C}$ in the dark prior to transfer into a growth cabinet. Plants were grown on vertical ${ }^{1 / 2} \mathrm{MS}$ plates at $22^{\circ} \mathrm{C}$ under long day conditions $(16 \mathrm{~h}$ light- $8 \mathrm{~h}$ dark) for 8 to 11 days.

Western blotting. Proteins were transferred to nitrocellulose membrane (GE Healthcare, Amersham ${ }^{\mathrm{TM}}$ Highbond ${ }^{\mathrm{TM}}$-ECL) via wet western blotting at $4{ }^{\circ} \mathrm{C}$ and $30 \mathrm{~V}$ overnight. Membranes were blocked in TBS-Tween (0.1\%)-Milk (5\%) for $1 \mathrm{~h}$ at room temperature. For mCherry detection, membranes were incubated overnight with an anti-mCherry antibody (ab167453, dilution $1: 2000$; Abcam) followed by $1 \mathrm{~h}$ incubation with an anti-rabbit-horseradish peroxidase (HRP) antibody (dilution $1: 10,000$, Calbiochem). For GFP detection, membranes were incubated overnight with an anti-GFP-HRP antibody (130-091-833, dilution 1: 1000 , Miltenyi Biotec). For FLAG detection, membranes were incubated overnight with an anti-FLAG-HRP antibody (A8692, dilution 1:1000, Sigma). For SPX1 detection, membranes were incubated overnight with an anti-SPX1 antibody (dilution 1:1000, kind gift of Professor Mingguang Lei) followed by one hour incubation with an anti-rabbit-HRP antibody (dilution $1: 10,000$, Calbiochem). Antibodies were diluted in TBS-Tween (0.05\%)-Milk (2.5\%). Membranes were detected with SuperSignal ${ }^{\text {mot }}$ West Femto Maximum Sensitivity Substrate (34095, Thermo Scientific $c^{\mathrm{s}}$ ).

Determination of cellular Pi concentrations. To determine cellular Pi concentration at seedling stage, plants were transferred from ${ }^{1 / 2} \mathrm{MS}$ plates to $-\mathrm{Pi}^{1 / 2} \mathrm{MS}$ plates containing $1(\mathrm{w} / \mathrm{v}) \%$ sucrose and $0.8(\mathrm{w} / \mathrm{v}) \%$ agarose supplemented with either 0,1 , or $10 \mathrm{mM} \mathrm{Pi}\left(\mathrm{KH}_{2} \mathrm{PO}_{4} / \mathrm{K}_{2} \mathrm{HPO}_{4} \mathrm{pH}\right.$ 5.7) at $7 \mathrm{DAG}$, and grown at $22^{\circ} \mathrm{C}$ under long day conditions. At $14 \mathrm{DAG}$, seedlings were weighted and harvested into $1.5 \mathrm{~mL}$ tubes containing $500 \mu \mathrm{L}$ nanopure $\mathrm{H}_{2} \mathrm{O}$. Samples were frozen at $-80^{\circ} \mathrm{C}$ overnight, thawed at $80^{\circ} \mathrm{C}$ for $10 \mathrm{~min}$, refrozen at $-80^{\circ} \mathrm{C}$, incubated at $80^{\circ} \mathrm{C}$ in a thermomixer shaking at 1400 r.p.m. for $1 \mathrm{~h}$, and briefly centrifuged to sediment plant tissue. Pi content was measured by the colorimetric molybdate assay ${ }^{60}$. In brief, $600 \mu \mathrm{L}$ ammonium molybdate solution $(0.44 \mathrm{~g}$ of ammonium molybdate tetra hydrate in $97.3 \mathrm{~mL}$ nanopure water; add $2.66 \mathrm{~mL}$ concentrated $(18 \mathrm{M}) \mathrm{H}_{2} \mathrm{SO}_{4}$ to a final volume of $100 \mathrm{~mL}), 100 \mu \mathrm{L} 10 \%$ ascorbic acid, and $300 \mu \mathrm{L}$ sample $(250 \mu \mathrm{L}$ nanopure water $+50 \mu \mathrm{L}$ extracts) or $300 \mu \mathrm{L} \mathrm{NaPi}$ standard solution were mixed. Samples were incubated at $37^{\circ} \mathrm{C}$ for $1 \mathrm{~h}$ and absorbance at $820 \mathrm{~nm}$ was measured.
RNA analyses. At 14 DAG, 50-150 mg seedlings were harvested in $2 \mathrm{ml}$ Eppendorf tubes containing two metal beads each, shock-frozen in liquid nitrogen and ground in a tissue lyzer (MM400, Retsch). RNA extraction was performed using the ReliaPrep RNA Tissue Miniprep System (Promega) including in column DNaseI treatment to remove genomic DNA. First strand cDNA synthesis was performed from 1 to $2.5 \mu \mathrm{g}$ of total RNA using Superscript II RT (Invitrogen) with oligo(dT) primers. Quantitative reverse transcriptase PCR (qRT-PCR) was performed in $10 \mu \mathrm{L}$ reactions containing $1 \times$ SYBR-Green fluorescent stain (Applied Biosystems) and measured using a 7900HT Fast Real Time PCR-System (Applied Biosystems). qRT-PCR program: $2^{\prime}-95^{\circ} \mathrm{C} ; 40 \times\left(300^{\prime \prime}-95^{\circ} \mathrm{C} ; 30 "-60^{\circ} \mathrm{C}\right.$; $20^{\prime \prime}-72^{\circ} \mathrm{C}$ ); melting curve $95^{\circ} \mathrm{C}-60^{\circ} \mathrm{C}-95^{\circ} \mathrm{C}$. A primer list can be found in Supplementary Table 3e. Expression levels of target genes were normalized against the housekeeping gene Actin2. For every genotype, three biological replicates were analyzed in technical triplicates.

Transient transformation of $\boldsymbol{N}$. benthamiana. For each construct, $4 \mathrm{ml}$ of $A$. tumefaciens strain pGV2260 suspension culture were grown overnight at $28^{\circ} \mathrm{C}$. Cells were collected by centrifugation at $700 \times g$ for $15 \mathrm{~min}$ and resuspended in transformation buffer $(10 \mathrm{mM} \mathrm{MgCl} 2,10 \mathrm{mM}$ MES pH 5.6, $150 \mu \mathrm{M}$ acetosyringone). Cell density was measured and set to a final $\mathrm{OD}_{600 \mathrm{~nm}}$ of 0.5 for SPX1 and PHR1, and to 0.1 for the silencing suppressor P19. Suspension cultures were incubated for $2 \mathrm{~h}$ in the dark at room temperature and subsequently mixed at a volume ratio of $1: 1: 1$ (SPX1: PHR1:P19). N. benthamiana leaves were infiltrated using a $0.5 \mathrm{ml}$ syringe and 3 leaf disks $(d=1 \mathrm{~cm})$ per sample were harvested after 3 days, snap-frozen in liquid nitrogen, and stored at $-80^{\circ} \mathrm{C}$.

Co-immunoprecipitation. For co-immunoprecipitation experiments with proteins transiently expressed in N. benthamiana, samples were ground in liquid nitrogen with plastic mortars and proteins were extracted with $600 \mu \mathrm{L}$ of homogenization buffer $(50 \mathrm{mM}$ Tris-HCl pH 7.5, $150 \mathrm{mM} \mathrm{NaCl}, 0.25 \%$ Triton X-100, 5\% (v/v) glycerol, $1 \mathrm{mM}$ phenylmethylsulfonyl fluoride, cOmplete ${ }^{\mathrm{TM}}$ EDTA-free protease inhibitor cocktail (Roche). Samples were incubated at $4{ }^{\circ} \mathrm{C}$ for $10 \mathrm{~min}$ with gently rotation and subsequently centrifuged at $16,000 \times g$ and $4^{\circ} \mathrm{C}$ for $15 \mathrm{~min}$. Supernatants were transferred to fresh tubes and further centrifuged at $16,000 \times g$ and $4{ }^{\circ} \mathrm{C}$ for $15 \mathrm{~min}$. Supernatants were transferred to fresh tubes, while $50 \mu \mathrm{L}$ of each supernatant were taken and mixed with $10 \mu \mathrm{L} 6 \times$ SDS sample buffer (input), the remaining supernatants were mixed with $50 \mu \mathrm{L}$ magnetic $\mu$ MACS anti-GFP beads (Miltenyi Biotec) and incubated at $4{ }^{\circ} \mathrm{C}$ for $2 \mathrm{~h}$ with gently rotation. MACS columns (Miltenyi Biotec) were used with a $\mu$ MACS Separator (Milteyi Biotec). MACS columns were washed with $200 \mu \mathrm{L}$ of homogenization buffer and samples were loaded. Columns were washed either four times with $200 \mu \mathrm{L}$ of homogenization buffer and once with wash buffer 2 (Miltenyi Biotec), or three times with $200 \mu \mathrm{L}$ of homogenization buffer, three times with wash buffer 1 (Miltenyi Biotec) and once with wash buffer 2 . Columns were incubated with $20 \mu \mathrm{L}$ preheated elution buffer (Miltenyi Biotec) for $5 \mathrm{~min}$ at room temperature. Elution buffer $(50 \mu \mathrm{L})$ was added and eluates were recovered. Inputs and eluates were boiled for $5 \mathrm{~min}$ at $95^{\circ} \mathrm{C}$ prior and separated on 9\% SDS-polyacrylamide electrophoresis gels. Coimmunoprecipitation experiments for proteins stably or natively expressed in $A$. thaliana were performed as previously described ${ }^{29}$

Statistics. All statistical analyses and data plots have been performed and generated with $\mathrm{R}$ version 3.5.2 $2^{61}$ and the packages "Hmisc ${ }^{62}$," "agricolae ${ }^{63}$," "car ${ }^{64}$," "multcompView 65 ," and "multcomp $p^{66}$." qRT-PCR data were power transformed with the Box-Cox transformation and a significant one-way analysis of variance (ANOVA) followed by a Dunnett's post hoc test was performed for multiple comparisons of several genotypes vs. wild type (Col-0) shown in Supplementary Fig. 8a. Pi content was analyzed with a one-way ANOVA followed by a Dunnett's post hoc test for multiple comparisons of several genotypes vs. wild type (Col-0) shown in Fig. 4 b and Supplementary Fig. $8 \mathrm{~b}-$ d. $\left({ }^{*} p<0.5 ;{ }^{* *} p<0.01 ;{ }^{* * *} p<0.001\right)$

Reporting summary. Further information on research design is available in the Nature Research Reporting Summary linked to this article.

\section{Data availability}

Data supporting the findings of this manuscript are available from the corresponding authors upon reasonable request. A reporting summary for this article is available as a Supplementary Information file. Coordinates and structure factors have been deposited in the Protein Data Bank (PDB) with accession codes 6TO5 (form1), $6 \mathrm{TO}$ (form2), and 6TOC (form 3). The associated X-ray diffraction images and data processing files have been deposited at http://zenodo.org with DOIs https://doi.org/10.5281/zenodo.3570698 (form1), https://doi.org/10.5281/zenodo.3570977 (form2), and https://doi.org/10.5281/ zenodo.3571040 (form 3). Source data are provided with this paper.

Received: 13 December 2019; Accepted: 9 December 2020; Published online: 15 January 2021 


\section{References}

1. Wykoff, D. D., Grossman, A. R., Weeks, D. P., Usuda, H. \& Shimogawara, K. Psr1, a nuclear localized protein that regulates phosphorus metabolism in Chlamydomonas. Proc. Natl Acad. Sci. USA 96, 15336-15341 (1999).

2. Rubio, V. et al. A conserved MYB transcription factor involved in phosphate starvation signaling both in vascular plants and in unicellular algae. Genes Dev. 15, 2122-2133 (2001).

3. Riechmann, J. L. et al. Arabidopsis transcription factors: genome-wide comparative analysis among eukaryotes. Science 290, 2105-2110 (2000).

4. Zhou, J. et al. OsPHR2 is involved in phosphate-starvation signaling and excessive phosphate accumulation in shoots of plants. Plant Physiol. 146, 1673-1686 (2008).

5. Ren, F. et al. Brassica napus PHR1 gene encoding a MYB-like protein functions in response to phosphate starvation. PLoS ONE 7, e44005 (2012).

6. Wang, J. et al. A phosphate starvation response regulator Ta-PHR1 is involved in phosphate signalling and increases grain yield in wheat. Ann. Bot. 111, 1139-1153 (2013).

7. Bustos, R. et al. A central regulatory system largely controls transcriptional activation and repression responses to phosphate starvation in Arabidopsis. PLoS Genet. 6, e1001102 (2010).

8. Nilsson, L., Müller, R. \& Nielsen, T. H. Increased expression of the MYBrelated transcription factor, PHR1, leads to enhanced phosphate uptake in Arabidopsis thaliana. Plant Cell Environ. 30, 1499-1512 (2007).

9. Pant, B. D. et al. The transcription factor PHR1 regulates lipid remodeling and triacylglycerol accumulation in Arabidopsis thaliana during phosphorus starvation. J. Exp. Bot. 66, 1907-1918 (2015).

10. Bari, R., Datt Pant, B., Stitt, M. \& Scheible, W.-R. PHO2, microRNA399, and PHR1 define a phosphate-signaling pathway in plants. Plant Physiol. 141, 988-999 (2006).

11. Rouached, H., Secco, D., Arpat, B. \& Poirier, Y. The transcription factor PHR1 plays a key role in the regulation of sulfate shoot-to-root flux upon phosphate starvation in Arabidopsis. BMC Plant Biol. https://doi.org/ 10.1186/1471-2229-11-19 (2011).

12. Khan, G. A. et al. Coordination between zinc and phosphate homeostasis involves the transcription factor PHR1, the phosphate exporter PHO1, and its homologue $\mathrm{PHO} 1 ; \mathrm{H} 3$ in Arabidopsis. J. Exp. Bot. 65, 871-884 (2014).

13. Nilsson, L., Lundmark, M., Jensen, P. E. \& Nielsen, T. H. The Arabidopsis transcription factor PHR1 is essential for adaptation to high light and retaining functional photosynthesis during phosphate starvation. Physiol. Plant 144, 35-47 (2012).

14. Bournier, M. et al. Arabidopsis ferritin 1 (AtFer1) gene regulation by the phosphate starvation response 1 (AtPHR1) transcription factor reveals a direct molecular link between iron and phosphate homeostasis. J. Biol. Chem. 288, 22670-22680 (2013)

15. Castrillo, G. et al. Root microbiota drive direct integration of phosphate stress and immunity. Nature 543, 513-518 (2017).

16. Liu, F. et al. OsSPX1 suppresses the function of OsPHR2 in the regulation of expression of OsPT2 and phosphate homeostasis in shoots of rice. Plant J. 62, 508-517 (2010).

17. Shi, J. et al. The paralogous SPX3 and SPX5 genes redundantly modulate Pi homeostasis in rice. J. Exp. Bot. 65, 859-870 (2014).

18. Wang, Z. et al. Rice SPX1 and SPX2 inhibit phosphate starvation responses through interacting with PHR2 in a phosphate-dependent manner. Proc. Natl Acad. Sci. USA 111, 14953-14958 (2014).

19. Puga, M. I. et al. SPX1 is a phosphate-dependent inhibitor of phosphate starvation response 1 in Arabidopsis. Proc. Natl Acad. Sci. USA 111, 14947-14952 (2014)

20. Duan, K. et al. Characterization of a sub-family of Arabidopsis genes with the SPX domain reveals their diverse functions in plant tolerance to phosphorus starvation. Plant J. 54, 965-975 (2008).

21. Wang, C. et al. Involvement of OsSPX1 in phosphate homeostasis in rice. Plant J. 57, 895-904 (2009).

22. Wang, Z. et al. Regulation of OsSPX1 and OsSPX3 on expression of OsSPX domain genes and Pi-starvation signaling in rice. J. Integr. Plant Biol. 51 663-674 (2009).

23. Lv, Q. et al. SPX4 negatively regulates phosphate signaling and homeostasis through its interaction with PHR2 in rice. Plant Cell 26, 1586-1597 (2014).

24. Zhong, Y. et al. Rice SPX6 negatively regulates the phosphate starvation response through suppression of the transcription factor PHR2. N. Phytol. 219, 135-148 (2018).

25. Osorio, M. B. et al. SPX4 acts on PHR1-dependent and -independent regulation of shoot phosphorus status in Arabidopsis. Plant Physiol. 181, 332-352 (2019).

26. Qi, W., Manfield, I. W., Muench, S. P. \& Baker, A. AtSPX1 affects the AtPHR1-DNA-binding equilibrium by binding monomeric AtPHR1 in solution. Biochem. J. 474, 3675-3687 (2017).
27. Ruan, W. et al. Two RING-finger ubiquitin E3 ligases regulate the degradation of SPX4, an internal phosphate sensor, for phosphate homeostasis and signaling in rice. Mol. Plant 12, 1060-1074 (2019).

28. Jung, J.-Y., Ried, M. K., Hothorn, M. \& Poirier, Y. Control of plant phosphate homeostasis by inositol pyrophosphates and the SPX domain. Curr. Opin. Biotechnol. 49, 156-162 (2018).

29. Dong, J. et al. Inositol pyrophosphate InsP8 acts as an intracellular phosphate signal in Arabidopsis. Mol. Plant 12, 1463-1473 (2019).

30. Wild, R. et al. Control of eukaryotic phosphate homeostasis by inositol polyphosphate sensor domains. Science 352, 986-990 (2016).

31. Shears, S. B. Intimate connections: Inositol pyrophosphates at the interface of metabolic regulation and cell signaling. J. Cell. Physiol. 233, 1897-1912 (2018)

32. Laha, D. et al. Arabidopsis ITPK1 and ITPK2 have an evolutionarily conserved phytic acid kinase activity. ACS Chem. Biol. 14, 2127-2133 (2019).

33. Desai, M. et al. Two inositol hexakisphosphate kinases drive inositol pyrophosphate synthesis in plants. Plant J. 80, 642-653 (2014).

34. Laha, D. et al. VIH2 regulates the synthesis of inositol pyrophosphate InsP8 and jasmonate-dependent defenses in Arabidopsis. Plant Cell 27, 1082-1097 (2015).

35. Zhu, J. et al. Two bifunctional inositol pyrophosphate kinases/phosphatases control plant phosphate homeostasis. eLife 8, e43582 (2019).

36. Couso, I. et al. Synergism between inositol polyphosphates and TOR kinase signaling in nutrient sensing, growth control, and lipid metabolism in Chlamydomonas. Plant Cell 28, 2026-2042 (2016).

37. $\mathrm{Gu}, \mathrm{C}$. et al. The significance of the bifunctional kinase/phosphatase activities of diphosphoinositol pentakisphosphate kinases (PPIP5Ks) for coupling inositol pyrophosphate cell signaling to cellular phosphate homeostasis. J. Biol. Chem. 292, 4544-4555 (2017).

38. Pascual-Ortiz, M. et al. Asp1 bifunctional activity modulates spindle function via controlling cellular inositol pyrophosphate levels in Schizosaccharomyces pombe. Mol. Cell. Biol. 38 (2018).

39. Jiang, M. et al. Structural basis for the Target DNA recognition and binding by the MYB domain of phosphate starvation response 1. FEBS J. 286, 2809-2821 (2019).

40. Draškovič, P. et al. Inositol hexakisphosphate kinase products contain diphosphate and triphosphate groups. Chem. Biol. 15, 274-286 (2008).

41. Mulugu, S. et al. A conserved family of enzymes that phosphorylate inositol hexakisphosphate. Science 316, 106-109 (2007).

42. Jaillais, Y. et al. Tyrosine phosphorylation controls brassinosteroid receptor activation by triggering membrane release of its kinase inhibitor. Genes Dev. 25, 232-237 (2011).

43. Holm, L. \& Sander, C. Protein structure comparison by alignment of distance matrices. J. Mol. Biol. 233, 123-138 (1993).

44. Andres, S. N. et al. Tetrameric Ctp1 coordinates DNA binding and DNA bridging in DNA double-strand-break repair. Nat. Struct. Mol. Biol. 22, 158-166 (2015).

45. Krissinel, E. \& Henrick, K. Inference of macromolecular assemblies from crystalline state. J. Mol. Biol. 372, 774-797 (2007).

46. Clough, S. J. \& Bent, A. F. Floral dip: a simplified method forAgrobacteriummediated transformation of Arabidopsis thaliana. Plant J. 16, 735-743 (1998).

47. Puschmann, R., Harmel, R. K. \& Fiedler, D. Scalable chemoenzymatic synthesis of inositol pyrophosphates. Biochemistry 58, 3927-3932 (2019).

48. Kabsch, W. Automatic processing of rotation diffraction data from crystals of initially unknown symmetry and cell constants. J. Appl. Crystallogr. 26, 795-800 (1993).

49. McCoy, A. J. et al. Phaser crystallographic software. J. Appl. Crystallogr. 40, 658-674 (2007)

50. Emsley, P. \& Cowtan, K. Coot: model-building tools for molecular graphics. Acta Crystallogr. D. Biol. Crystallogr. 60, 2126-2132 (2004).

51. Adams, P. D. et al. PHENIX: a comprehensive Python-based system for macromolecular structure solution. Acta Crystallogr. D. Biol. Crystallogr. 66, 213-221 (2010).

52. Murshudov, G. N., Vagin, A. A. \& Dodson, E. J. Refinement of macromolecular structures by the maximum-likelihood method. Acta Crystallogr. D. Biol. Crystallogr. 53, 240-255 (1997).

53. Davis, I. W. et al. MolProbity: all-atom contacts and structure validation for proteins and nucleic acids. Nucleic Acids Res. 35, W375-W383 (2007).

54. Goddard, T. D. et al. UCSF ChimeraX: meeting modern challenges in visualization and analysis. Protein Sci. 27, 14-25 (2018).

55. Bordoli, L. et al. Protein structure homology modeling using SWISS-MODEL workspace. Nat. Protoc. 4, 1-13 (2009).

56. Notredame, C., Higgins, D. G. \& Heringa, J. T-coffee: a novel method for fast and accurate multiple sequence alignment1. J. Mol. Biol. 302, 205-217 (2000).

57. Abriata, L. A. A simple spreadsheet program to simulate and analyze the farUV circular dichroism spectra of proteins. J. Chem. Educ. 88, 1268-1273 (2011).

58. Vranken, W. F. et al. The CCPN data model for NMR spectroscopy: development of a software pipeline. Proteins 59, 687-696 (2005). 
59. Murashige, T. \& Skoog, F. A revised medium for rapid growth and bio assays with tobacco tissue cultures. Physiol. Plant. 15, 473-497 (1962).

60. Ames, B. Assay of inorganic phosphate, total phosphate and phosphatases. Methods Enzym 8, 115-118 (1966).

61. R Core Team. R: A Language and Environment for Statistical Computing. https://www.R-project.org/ (R Foundation for Statistical Computing, Vienna, Austria, 2017).

62. Harrell Jr, F. E. Hmisc: Harrell Miscellaneous. https://CRAN.R-project.org/ package $=$ Hmisc (2020).

63. Mendiburu, F. agricolae: Statistical Procedures for Agricultural Research. $R$ package version 1.3-3. https://CRAN.R-project.org/package=agricolae (2020)

64. Fox, J. \& Weisberg, S. An $\{R\}$ Companion to Applied Regression. 3rd edn. https://socialsciences.mcmaster.ca/jfox/Books/Companion/ (Sage, Thousand Oaks, CA, 2019).

65. Graves, S. et al. multcompView: Visualizations of Paired Comparisons. $R$ package version 0.1-8. https://CRAN.R-project.org/package $=$ multcompView (2019).

66. Hothorn, T., Bretz, F. \& Westfall, P. Simultaneous inference in general parametric models. Biom. J. 50, 346-363 (2008).

\section{Acknowledgements}

This work was supported by European Research Council Consolidator Grant 818696/ INSPIRE (to M.H.), by Swiss National Foundation Sinergia Grant CRSII5_170925 (to M.H., S.H., and D.F.), and by an HHMI International Research Scholar Award (to M.H.). M.K.R. was supported by an EMBO long-term fellowship (ALTF-129-2017). We thank Irene Sabater for providing LI SPX1 and members of the Hothorn lab for critically reading the manuscript.

\section{Author contributions}

M.K.R.: Conceptualization, data curation, formal analysis, validation, investigation, visualization, methodology, and writing (original draft, review, and editing). R.W.: Conceptualization, data curation, formal analysis, validation, investigation, methodology, and writing (review and editing). J.Z.: Investigation, methodology, and writing (review and editing). J.P.: Conceptualization, data curation, formal analysis, validation, investigation, methodology, and writing (review and editing). K.S.: investigation, formal analysis, and methodology. L.B.: Investigation and methodology. R.K.H.: Methodology. L.A.A.: formal analysis and methodology. L.A.H.: Software, formal analysis, and methodology. D.F.: Resources, methodology, and writing (review and editing). S.H.: Resources, methodology, supervision, and writing (review and editing). M.H.: Conceptualization, resources, data curation, formal analysis, supervision, funding acquisition, validation, investigation, visualization, methodology, project administration, and writing (original draft, review, and editing).

\section{Competing interests}

The authors declare no competing interests.

\section{Additional information}

Supplementary information is available for this paper at https://doi.org/10.1038/s41467020-20681-4.

Correspondence and requests for materials should be addressed to M.H.

Peer review information Nature Communications thanks Aiwu Dong and the other, anonymous, reviewer(s) for their contribution to the peer review of this work. Peer review reports are available.

Reprints and permission information is available at http://www.nature.com/reprints

Publisher's note Springer Nature remains neutral with regard to jurisdictional claims in published maps and institutional affiliations.

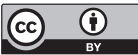

Open Access This article is licensed under a Creative Commons Attribution 4.0 International License, which permits use, sharing, adaptation, distribution and reproduction in any medium or format, as long as you give appropriate credit to the original author(s) and the source, provide a link to the Creative Commons license, and indicate if changes were made. The images or other third party material in this article are included in the article's Creative Commons license, unless indicated otherwise in a credit line to the material. If material is not included in the article's Creative Commons license and your intended use is not permitted by statutory regulation or exceeds the permitted use, you will need to obtain permission directly from the copyright holder. To view a copy of this license, visit http://creativecommons.org/ licenses/by/4.0/.

(C) The Author(s) 2021 